\title{
Asymptotic Behavior of Stochastic Strongly Wave Equation on Unbounded Domains
}

\author{
Zhaojuan Wang1, Shengfan Zhou ${ }^{2}$ \\ ${ }^{1}$ School of Mathematical Science, Huaiyin Normal University, Huaian, China \\ ${ }^{2}$ Department of Mathematics, Zhejiang Normal University, Jinhua, China \\ Email: wangzhaojuan2006@163.com, zhoushengfan@yahoo.com
}

Received 15 January 2015; accepted 25 March 2015; published 30 March 2015

Copyright (C) 2015 by authors and Scientific Research Publishing Inc.

This work is licensed under the Creative Commons Attribution International License (CC BY).

http://creativecommons.org/licenses/by/4.0/

c) (i) Open Access

\section{Abstract}

We study the asymptotic behavior of solutions to the stochastic strongly damped wave equation with additive noise defined on unbounded domains. We first prove the uniform estimates of solutions, and then establish the existence of a random attractor.

\section{Keywords}

\section{Stochastic Strongly Damped Wave Equation, Random Dynamical System, Random Attractor}

\section{Introduction}

Let $(\Omega, \mathcal{F}, \mathbb{P})$ be a probability space, where

$$
\Omega=\left\{\omega=\left(\omega_{1}, \omega_{2}, \cdots, \omega_{m}\right) \in C\left(\mathbb{R}, \mathbb{R}^{m}\right): \omega(0)=0\right\}
$$

the Borel $\sigma$-algebra $\mathcal{F}$ on $\Omega$ is generated by the compact open topology (see [1]), and $\mathbb{P}$ is the corresponding Wiener measure on $\mathcal{F}$. Define $\left(\theta_{t}\right)_{t \in \mathbb{R}}$ on $\Omega$ via

$$
\theta_{t} \omega(\cdot)=\omega(\cdot+t)-\omega(t), \quad t \in \mathbb{R}
$$

Thus, $\left(\Omega, \mathcal{F}, \mathbb{P},\left(\theta_{t}\right)_{t \in \mathbb{R}}\right)$ is an ergodic metric dynamical system.

Consider the following stochastic strongly damped wave equation with additive noise defined in the entire space $\mathbb{R}^{n}(n \leq 3)$ :

$$
u_{t t}-\Delta u_{t}+\alpha u_{t}-\Delta u+\lambda u+f(x, u)=g(x)+\sum_{j=1}^{m} h_{j}(x) \frac{\mathrm{d} W_{j}}{\mathrm{~d} t}
$$


with the initial value conditions

$$
u(x, 0)=u_{0}(x), \quad u_{t}(x, 0)=u_{1}(x), \quad x \in \mathbb{R}^{n}
$$

where $\Delta$ is the Laplacian with respect to the variable $x \in \mathbb{R}^{n}, u=u(x, t)$ is a real function of $x \in \mathbb{R}^{n}$ and $t \geqslant 0 ; \quad \alpha, \lambda$ are positive constants, $g \in L^{2}\left(\mathbb{R}^{n}\right)$ and $h_{j} \in H^{1}\left(\mathbb{R}^{n}\right) \quad(1 \leqslant j \leqslant m)$ are given; $f$ is a nonlinear function satisfying certain dissipative and growth conditions, and $\left\{W_{j}\right\}_{j=1}^{m}$ are independent two-sided realvalued Wiener processes on $(\Omega, \mathcal{F}, \mathbb{P})$. We identify $\omega(t)$ with $\left(W_{1}(t), W_{2}(t), \cdots, W_{m}(t)\right)$, i.e.,

$$
\omega(t)=\left(W_{1}(t), W_{2}(t), \cdots, W_{m}(t)\right), \quad t \in \mathbb{R}
$$

Many works have been done regarding the dynamics of a variety of systems related to Equation (1). For example, the asymptotical behavior of solutions for deterministic strongly damped wave equation has been studied by many authors (see [2]-[11], etc.). For stochastic wave equation, the asymptotical behavior of solutions have been studied by several authors (see [12]-[25], etc.). However, no results have been presented on random attractors for stochastic strongly damped wave equation (1) with additive noise on unbounded domains to date.

In general, the existence of global random attractor depends on some kind compactness (see, e.g., [26]-[30]). For Cauchy problem, the main question is how to overcome the difficulty of lacking the compactness of Sobolev embedding in unbounded domains. For some deterministic equations, the difficulty caused by the unboundedness of domains can be overcome by the energy equation approach. The energy equation method was developed by Ball in [31] [32] and used by many authors (see, e.g., [33]-[39]). Under certain circumstances, the tail-estimates method can be used to deal with the problem caused by the unboundedness of domains (see [40]). In this paper, we will combine the splitting technique in [20] with the idea of uniform estimates on the tails of solutions to investigate the existence of global attractor of the stochastic strongly damped wave Equation (1) defined on unbounded domains. The rest of this paper is organized as follows. In the next section, we recall some basic concepts related to random attractor for general random dynamical systems. In Section 3, we provide some basic settings about Equation (1) and show that it generates a random dynamical system, and then we prove the uniform estimates of solutions and obtain the existence of a random attractor for Equation (1).

Throughout this paper, we use $\|\cdot\|$ and $(\cdot, \cdot)$ to denote the norm and the inner product of $L^{2}\left(\mathbb{R}^{n}\right)$, respectively. The norm of a Banach space $X$ is generally written as \|\|$_{X}$. The symbol $C$ is a positive constant which may change its value from line to line.

\section{Preliminaries}

In this section, we collect some basic knowledge about general random dynamical systems (see [1] [41] for details). Let $\left(X,\|\cdot\|_{X}\right)$ be a separable Hilbert space with Borel $\sigma$-algebra $\mathcal{B}(X)$. Let $\left(\Omega, \mathcal{F}, \mathbb{P},\left(\theta_{t}\right)_{t \in \mathbb{R}}\right)$ be the metric dynamical system on the probability space $(\Omega, \mathcal{F}, \mathbb{P})$.

In the following, a property holds for $\mathbb{P}$-a.e. $\omega \in \Omega$ means that there is $\Omega_{0} \in \Omega$ with $\mathbb{P}\left(\Omega_{0}\right)=1$ and $\theta_{t} \Omega_{0}=\Omega_{0}$ for $t \in \mathbb{R}$.

Definition 1 A continuous random dynamical system on $X$ over $\left(\Omega, \mathcal{F}, \mathbb{P},\left(\theta_{t}\right)_{t \in \mathbb{R}}\right)$ is a $\left(\mathcal{B}\left(\mathbb{R}^{+}\right) \times \mathcal{F} \times \mathcal{B}(X), \mathcal{B}(X)\right)$-measurable mapping

$$
\varphi: \mathbb{R}^{+} \times \Omega \times X \rightarrow X, \quad(t, \omega, u) \mapsto \varphi(t, \omega, u)
$$

such that the following properties hold

- $\varphi(0, \omega, \cdot)$ is the identity on $X$;

- $\varphi(t+s, \omega, \cdot)=\varphi\left(t, \theta_{s} \omega, \varphi(s, \omega, \cdot)\right)$ for all $s, t \geqslant 0$;

- $\varphi(t, \omega, \cdot): X \rightarrow X$ is continuous for all $t \geqslant 0$.

Definition 2 (See [41])

- A set-valued mapping $\{D(\omega)\}: \Omega \rightarrow 2^{X} \backslash \varnothing, \omega \rightarrow D(\omega)$, is said to be a random set if the mapping $\omega \mapsto d(u, D(\omega))$ is measurable for any $u \in X$. If $D(\omega)$ is also closed (compact) for each $\omega \in \Omega$, $\{D(\omega)\}$ is called a random closed (compact) set. A random set $\{D(\omega)\}$ is said to be bounded if there exist $u_{0} \in X$ and a random variable $R(\omega)>0$ such that

$$
D(\omega) \subset\left\{u \in X:\left\|u-u_{0}\right\|_{X} \leqslant R(\omega)\right\} \text { for all } \omega \in \Omega
$$


- A random set $\{D(\omega)\}$ is called tempered provided for $\mathbb{P}$-a.e. $\omega \in \Omega$,

$$
\lim _{t \rightarrow+\infty} \mathrm{e}^{-\beta t} d\left(D\left(\theta_{-t} \omega\right)\right)=0 \quad \text { for all } \beta>0
$$

where $d(D)=\sup \left\{\|b\|_{X}: b \in D\right\}$.

Let $\Rightarrow$ be the set of all random tempered sets in $X$.

- A random set $\{B(\omega)\}$ is said to be a random absorbing set if for any tempered random set $\{D(\omega)\}$, and $\mathbb{P}$-a.e. $\omega \in \Omega$, there exists $t_{0}(\omega)$ such that

$$
\varphi\left(t, \theta_{-t} \omega, D\left(\theta_{-t} \omega\right)\right) \subset B(\omega) \text { for all } t \geqslant t_{0}(\omega)
$$

- A random set $\left\{B_{1}(\omega)\right\}$ is said to be a random attracting set if for any tempered random set $\{D(\omega)\}$, and $\mathbb{P}$-a.e. $\omega \in \Omega$, we have

$$
\lim _{t \rightarrow+\infty} d_{H}\left(\varphi\left(t, \theta_{-t} \omega, D\left(\theta_{-t} \omega\right)\right), B_{1}(\omega)\right)=0
$$

where $d_{H}$ is the Hausdorff semi-distance given by $d_{H}(E, F)=\sup _{u \in E} \inf _{v \in F}\|u-v\|_{X}$ for any $E, F \subset X$.

- $\varphi$ is said to be asymptotically compact in $X$ if for $\mathbb{P}$-a.e. $\omega \in \Omega,\left\{\varphi\left(t_{n}, \theta_{-t_{n}} \omega, x_{n}\right)\right\}_{n=1}^{\infty}$ has a convergent subsequence in $X$ whenever $t_{n} \rightarrow+\infty$, and $x_{n} \in B\left(\theta_{-t_{n}} \omega\right)$ with $\{B(\omega)\} \in \mathscr{P}$.

- A random compact set $\{A(\omega)\}$ is said to be a random attractor if it is a random attracting set and $\varphi(t, \omega, A(\omega))=A\left(\theta_{t} \omega\right)$ for $\mathbb{P}$-a.e. $\omega \in \Omega$ and all $t \geqslant 0$.

Theorem 1 (See [41]) Let $\varphi$ be a continuous random dynamical system with state space $X$ over $\left(\Omega, \mathcal{F}, \mathbb{P},\left(\theta_{t}\right)_{t \in \mathbb{R}}\right)$. If there is a closed random absorbing set $\{B(\omega)\}$ of $\varphi$ and $\varphi$ is asymptotically compact in $X$, then $\{A(\omega)\}$ is a random attractor of $\varphi$, where

$$
A(\omega)=\bigcap_{t>0 \tau \geqslant t} \varphi\left(\tau, \theta_{-\tau} \omega, B\left(\theta_{-\tau} \omega\right)\right), \quad \omega \in \Omega
$$

Moreover, $\{A(\omega)\}$ is the unique random attractor of $\varphi$.

\section{Existence of Random Attractor}

\subsection{Basic Settings}

In this subsection, we outline some basic settings about (1)-(2) and show that it generates a random dynamical system.

Let $\xi=u_{t}+\sigma u$ where $\sigma$ is a small positive constant whose value will be determined later, then (1)-(2) can be rewritten as the equivalent system

$$
\left\{\begin{array}{l}
\frac{\mathrm{d} u}{\mathrm{~d} t}=\xi-\sigma u, \\
\frac{\mathrm{d} \xi}{\mathrm{d} t}=(\sigma-\alpha) \xi+\Delta \xi+(\sigma(\alpha-\sigma)-\lambda) u+(1-\sigma) \Delta u-f(x, u)+g(x)+\sum_{j=1}^{m} h_{j}(x) \frac{\mathrm{d} W_{j}}{\mathrm{~d} t},
\end{array}\right.
$$

with the initial value conditions

$$
u(x, 0)=u_{0}(x), \quad \xi(x, 0)=\xi_{0}(x)
$$

where $\xi_{0}(x)=u_{1}(x)+\sigma u_{0}(x), \quad x \in \mathbb{R}^{n}$.

Let $F(x, u)=\int_{0}^{u} f(x, s)$ ds for $x \in \mathbb{R}^{n}$ and $u \in \mathbb{R}$. The function $f$ will be assumed to satisfy the following conditions,

(F1) $|f(x, u)| \leqslant c_{1}|u|^{k}+\eta_{1}(x)$

(F2) $f(x, u) u-c_{2} F(x, u) \geqslant \eta_{2}(x)$

(F3) $F(x, u) \geqslant c_{3}|u|^{k+1}-\eta_{3}(x)$ 
(F4) $\left|f_{u}(x, u)\right| \leqslant c_{4}|u|^{k-1}+\eta_{4}(x)$

where $1 \leqslant k<\infty$ for $n=1,2$ and $1 \leqslant k<3$ for $n=3, \quad \eta_{1}(x) \in L^{2}(\mathbb{R}), \quad \eta_{2}(x) \in L^{1}(\mathbb{R}), \quad \eta_{3}(x) \in L^{1}(\mathbb{R})$ and $\eta_{4}(x) \in H^{1}(\mathbb{R}), \quad c_{i}(i=1,2,3,4)$ are positive constant. Note that (F1) and (F2) imply

$$
F(x, u) \leqslant c\left(|u|^{2}+|u|^{k+1}+\eta_{1}^{2}+\eta_{2}\right)
$$

For our purpose, it is convenient to convert the problem (3)-(4) (or (1)-(2)) into a deterministic system with a random parameter, and then show that it generates a random dynamical system.

Let $\left(\Omega, \mathcal{F}, \mathbb{P},\left(\theta_{t}\right)_{t \in \mathbb{R}}\right)$ be the ergodic metric dynamical system in Section 1 . For $j \in\{1,2, \cdots, m\}$, consider the one-dimensional Ornstein-Uhlenbeck equation

$$
\mathrm{d} z_{j}+z_{j} \mathrm{~d} t=\mathrm{d} W_{j}(t)
$$

Its unique stationary solution is given by

$$
z_{j}\left(\theta_{t} \omega_{j}\right)=-\int_{-\infty}^{0} \mathrm{e}^{s}\left(\theta_{t} \omega_{j}\right)(s) \mathrm{d} s, \quad t \in \mathbb{R}
$$

Note that the random variable $\left|z_{j}\left(\omega_{j}\right)\right|$ is tempered, and there is a $\theta_{t}$-invariant $\Omega_{0} \subset \Omega$ with $\mathbb{P}\left(\Omega_{0}\right)=1$ such that $t \mapsto z_{j}\left(\theta_{t} \omega_{j}\right)$ is continuous for $\omega \in \Omega_{0}$ and $j=1,2, \cdots, m$. Therefore, it follows from Proposition 4.3.3 in [1] that for any $\epsilon>0$, there exists a tempered function $\gamma(\omega)>0$ such that

$$
\sum_{j=1}^{m}\left(\left|z_{j}\left(\omega_{j}\right)\right|^{2}+\left|z_{j}\left(\omega_{j}\right)\right|^{k+1}\right) \leqslant \gamma(\omega)
$$

where $\gamma(\omega)$ satisfies, for $\mathbb{P}$-a.e. $\omega \in \Omega$,

$$
\gamma\left(\theta_{t} \omega\right) \leqslant \mathrm{e}^{\epsilon|t|} \gamma(\omega), \quad t \in \mathbb{R}
$$

Then it follows from the above, for $\mathbb{P}$-a.e. $\omega \in \Omega$,

$$
\sum_{j=1}^{m}\left(\left|z_{j}\left(\theta_{t} \omega_{j}\right)\right|^{2}+\left|z_{j}\left(\theta_{t} \omega_{j}\right)\right|^{k+1}\right) \leqslant \mathrm{e}^{\epsilon t \mid} \gamma(\omega), \quad t \in \mathbb{R}
$$

Put $z\left(\theta_{t} \omega\right)=\sum_{j=1}^{m} h_{j} z_{j}\left(\theta_{t} \omega_{j}\right)$, which solves $\mathrm{d} z+z \mathrm{~d} t=\sum_{j=1}^{m} h_{j} \mathrm{~d} W_{j}$.

Now, let $v(x, t)=\xi(x, t)-z\left(\theta_{t} \omega\right)$, we obtain the equivalent system of (3)-(4),

$$
\left\{\begin{array}{l}
\frac{\mathrm{d} u}{\mathrm{~d} t}=v-\sigma u+z\left(\theta_{t} \omega\right), \\
\frac{\mathrm{d} v}{\mathrm{~d} t}=(\sigma-\alpha) v+\Delta v-\left(\lambda+\sigma^{2}-\alpha \sigma\right) u+(1-\sigma) \Delta u-f(x, u)+g(x)+\Delta z\left(\theta_{t} \omega\right)+(1+\sigma-\alpha) z\left(\theta_{t} \omega\right),
\end{array}\right.
$$

with the initial value conditions

$$
u(x, 0)=u_{0}(x), \quad v(x, 0)=v_{0}(x)
$$

where $v_{0}(x)=\xi_{0}(x)-z(\omega), \quad x \in \mathbb{R}^{n}$. We will consider (9)-(10) for $\omega \in \tilde{\Omega}$ and write $\tilde{\Omega}$ as $\Omega$ from now on.

Let $E=H^{1}\left(\mathbb{R}^{n}\right) \times L^{2}\left(\mathbb{R}^{n}\right)$, endowed with the usual norm

$$
\|Y\|_{H^{1} \times L^{2}}=\left(\|\nabla u\|^{2}+\|u\|^{2}+\|v\|^{2}\right)^{\frac{1}{2}}, \text { for } Y=(u, v)^{\mathrm{T}} \in E
$$

where $\|\cdot\|$ denotes the usual norm in $L^{2}\left(\mathbb{R}^{n}\right)$ and $\mathrm{T}$ stands for the transposition.

By a standard method as in [2] [3] [42], one may show that under conditions (F1)-(F4), for $\left(u_{0}, v_{0}\right)^{\mathrm{T}} \in E$, problem (9)-(10) has a unique solution $\left(u\left(t, \omega, u_{0}\right), v\left(t, \omega, v_{0}\right)\right)^{\mathrm{T}}$ which is continuous with respect to $\left(u_{0}, v_{0}\right)^{\mathrm{T}}$ 
in $E$ for all $t>0$. Hence, the solution mapping

$$
\bar{S}_{\sigma}(t, \omega): \varphi_{0} \mapsto \varphi\left(t, \omega, \varphi_{0}\right)=\left(u\left(t, \omega, v_{0}\right), v\left(t, \omega, v_{0}\right)\right)^{\mathrm{T}}, \quad E \rightarrow E
$$

generates a continuous random dynamical system, where $\varphi_{0}=\left(u_{0}, v_{0}\right)^{\mathrm{T}}$. Introducing the homeomorphism $\mathcal{R}\left(\theta_{t} \omega\right)(u, \xi)^{\mathrm{T}}=\left(u, \xi+z\left(\theta_{t} \omega\right)\right)^{\mathrm{T}},(u, \xi)^{\mathrm{T}} \in E$ whose inverse homeomorphism

$$
\mathcal{R}^{-1}\left(\theta_{t} \omega\right)(u, \xi)^{\mathrm{T}}=\left(u, \xi-z\left(\theta_{t} \omega\right)\right)^{\mathrm{T}}
$$

Then, the transformation

$$
S_{\sigma}(t, \omega)=\mathcal{R}\left(\theta_{t} \omega\right) \bar{S}_{\sigma}(t, \omega) \mathcal{R}^{-1}\left(\theta_{t} \omega\right)
$$

also generates a random dynamical system associated with (3)-(4). Note that the two random dynamical systems are equivalent. By (13), it is easy to check that $S_{\sigma}(t, \omega)$ has a random attractor $\left\{\mathcal{R}\left(\theta_{t} \omega\right) \mathcal{A}(\omega) \mathcal{R}^{-1}\left(\theta_{t} \omega\right)\right\}$ provided $\bar{S}_{\sigma}(t, \omega)$ possesses a random attractor $\{\mathcal{A}(\omega)\}$. Then, we only need to consider the random dynamical system $\bar{S}_{\sigma}(t, \omega)$.

\subsection{Uniform Estimates of Solutions}

In this subsection, we derive uniform estimates on the solutions of the stochastic strongly damped wave Equations (3)-(4) defined on $\mathbb{R}^{n}$ when $t \rightarrow \infty$. These estimates are necessary for proving the existence of bounded absorbing sets and the asymptotic compactness of the random dynamical system associated with the equations. In particular, we will show that the tails of the solutions for large space variables are uniformly small when time is sufficiently large.

We assume that $\sigma$ is the collection of all tempered random subsets of $E$ from now on. Let $\sigma \in(0,1)$ be small enough such that

$$
\lambda+\sigma^{2}-\alpha \sigma>0, \quad \alpha-\sigma>0
$$

Set

$$
\delta=\min \left\{\alpha-\sigma, \sigma, \frac{c_{2} \sigma}{2}\right\}
$$

where $c_{2}$ is the positive constant in (F2).

We define a new norm $\|\cdot\|_{E}$ by

$$
\|Y\|_{E}=\left(\|v\|^{2}+\left(\lambda+\sigma^{2}-\alpha \sigma\right)\|u\|^{2}+(1-\sigma)\|\nabla u\|^{2}\right)^{\frac{1}{2}}
$$

for $Y=(u, v)^{\mathrm{T}} \in E$. It is easy to check that $\|\cdot\|_{E}$ is equivalent to the usual norm $\|\cdot\|_{H^{1} \times L^{2}}$ in (11).

The next lemma shows that $\bar{S}_{\sigma}(t, \omega)$ has an absorbing set in $\mathscr{V}$.

Lemma 1 Assume that (F1)-(F4), $g \in L^{2}\left(\mathbb{R}^{n}\right)$ and $h_{j} \in H^{1}\left(\mathbb{R}^{n}\right)(1 \leqslant j \leqslant m)$ hold. Then there exists a random ball $\left\{A_{0}(\omega)\right\} \in \mathcal{Q}$ centered at 0 with random radius $\rho(\omega)>0$ such that $\left\{A_{0}(\omega)\right\}$ is a random absorbing set for $\bar{S}_{\sigma}(t, \omega)$ in $\mathcal{Y}$, that is, for any $\{B(\omega)\} \in \mathcal{O}$ and $\mathbb{P}$-a.e. $\omega \in \Omega$, there is $T_{B}(\omega)>0$ such that

$$
\varphi\left(t, \theta_{-t} \omega, B\left(\theta_{-t} \omega\right)\right) \subseteq A_{0}(\omega) \text { for all } t>T_{B}(\omega)
$$

Proof. Taking the inner product of the second equation of (9) with $v$ in $L^{2}\left(\mathbb{R}^{n}\right)$, we find that

$$
\begin{aligned}
\frac{1}{2} \frac{\mathrm{d}}{\mathrm{d} t}\|v\|^{2}= & (\sigma-\alpha)\|v\|^{2}-\|\nabla v\|^{2}-\left(\lambda+\sigma^{2}-\alpha \sigma\right)(u, v)+(1-\sigma)(\Delta u, v)-(f(x, u), v)+(g(x), v) \\
& +\left(\Delta z\left(\theta_{t} \omega\right), v\right)+(1+\sigma-\alpha)\left(z\left(\theta_{t} \omega\right), v\right) .
\end{aligned}
$$


By the first equation of (9), we have

$$
v=\frac{\mathrm{d} u}{\mathrm{~d} t}+\sigma u-z\left(\theta_{t} \omega\right)
$$

Then substituting the above $v$ into the second and third terms on the left-hand side of (17), we find that

$$
\begin{aligned}
(u, v) & =\left(u, \frac{\mathrm{d} u}{\mathrm{~d} t}+\sigma u-z\left(\theta_{t} \omega\right)\right) \\
& =\frac{1}{2} \frac{\mathrm{d}}{\mathrm{d} t}\|u\|^{2}+\sigma\|u\|^{2}-\left(u, z\left(\theta_{t} \omega\right)\right) \\
& \geqslant \frac{1}{2} \frac{\mathrm{d}}{\mathrm{d} t}\|u\|^{2}+\frac{\sigma}{2}\|u\|^{2}-\frac{1}{2 \sigma}\left\|z\left(\theta_{t} \omega\right)\right\|^{2}, \\
(\Delta u, v) & =-\left(\nabla u, \nabla\left(\frac{\mathrm{d} u}{\mathrm{~d} t}+\sigma u-z\left(\theta_{t} \omega\right)\right)\right) \\
& =-\frac{1}{2} \frac{\mathrm{d}}{\mathrm{d} t}\|\nabla u\|^{2}-\sigma\|\nabla u\|^{2}+\left(\nabla u, \nabla z\left(\theta_{t} \omega\right)\right) \\
& \leqslant-\frac{1}{2} \frac{\mathrm{d}}{\mathrm{d} t}\|\nabla u\|^{2}-\frac{\sigma}{2}\|\nabla u\|^{2}+\frac{1}{2 \sigma}\left\|\nabla z\left(\theta_{t} \omega\right)\right\|^{2} .
\end{aligned}
$$

From conditions (F1)-(F3) we get

$$
\begin{aligned}
& -(f(x, u), v)=-\left(f(x, u), \frac{\mathrm{d} u}{\mathrm{~d} t}+\sigma u-z\left(\theta_{t} \omega\right)\right) \\
& =-\frac{\mathrm{d}}{\mathrm{d} t} \int_{\mathbb{R}^{n}} F(x, u) \mathrm{d} x-\sigma(f(x, u), u)+\left(f(x, u), z\left(\theta_{t} \omega\right)\right) \\
& \leqslant-\frac{\mathrm{d}}{\mathrm{d} t} \int_{\mathbb{R}^{n}} F(x, u) \mathrm{d} x-c_{2} \sigma \int_{\mathbb{R}^{n}} F(x, u) \mathrm{d} x-\sigma \int_{\mathbb{R}^{n}} \eta_{2}(x) \mathrm{d} x+\int_{\mathbb{R}^{n}}\left(c_{1}|u|^{k}+\eta_{1}(x)\right)\left|z\left(\theta_{t} \omega\right)\right| \mathrm{d} x \\
& \leqslant-\frac{\mathrm{d}}{\mathrm{d} t} \int_{\mathbb{R}^{n}} F(x, u) \mathrm{d} x-c_{2} \sigma \int_{\mathbb{R}^{n}} F(x, u) \mathrm{d} x-\sigma \int_{\mathbb{R}^{n}} \eta_{2}(x) \mathrm{d} x+c_{1}\left(\int_{\mathbb{R}^{n}}|u|^{k+1} \mathrm{~d} x\right)^{\frac{k}{k+1}}\left\|z\left(\theta_{t} \omega\right)\right\|_{L^{k+1}}+\left\|\eta_{1}(x)\right\| \cdot\left\|z\left(\theta_{t} \omega\right)\right\| \\
& \leqslant-\frac{\mathrm{d}}{\mathrm{d} t} \int_{\mathbb{R}^{n}} F(x, u) \mathrm{d} x-\frac{c_{2} \sigma}{2} \int_{\mathbb{R}^{n}} F(x, u) \mathrm{d} x-\sigma \int_{\mathbb{R}^{n}} \eta_{2}(x) \mathrm{d} x+\frac{c_{2} \sigma}{2} \int_{\mathbb{R}^{n}} \eta_{3}(x) \mathrm{d} x+c\left\|z\left(\theta_{t} \omega\right)\right\|_{H^{1}}^{k+1}+\left\|\eta_{1}(x)\right\| \cdot\left\|z\left(\theta_{t} \omega\right)\right\| .
\end{aligned}
$$

Using the Cauchy-Schwartz inequality and the Young inequality, we have

$$
\begin{aligned}
& (g(x), v) \leqslant\|g(x)\| \cdot\|v\| \leqslant \frac{\|g(x)\|^{2}}{\alpha-\sigma}+\frac{\alpha-\sigma}{4}\|v\|^{2} \\
& \left(\Delta z\left(\theta_{t} \omega\right), v\right)=-\left(\nabla z\left(\theta_{t} \omega\right), \nabla v\right) \leqslant\left\|\nabla z\left(\theta_{t} \omega\right)\right\| \cdot\|\nabla v\| \leqslant \frac{1}{4}\left\|\nabla z\left(\theta_{t} \omega\right)\right\|^{2}+\|\nabla v\|^{2} \\
& (1+\sigma-\alpha)\left(z\left(\theta_{t} \omega\right), v\right) \leqslant\left(\alpha-\sigma-2+\frac{1}{\alpha-\sigma}\right)\left\|z\left(\theta_{t} \omega\right)\right\|^{2}+\frac{\alpha-\sigma}{4}\|v\|^{2}
\end{aligned}
$$

By (19)-(24), it follows from (17) that

$$
\begin{aligned}
& \frac{\mathrm{d}}{\mathrm{d} t}\left(\|v\|^{2}+\left(\lambda+\sigma^{2}-\alpha \sigma\right)\|u\|^{2}+(1-\sigma)\|\nabla u\|^{2}+2 \int_{\mathbb{R}^{n}} F(x, u) \mathrm{d} x\right) \\
& \leqslant(\sigma-\alpha)\|v\|^{2}-\sigma\left(\lambda+\sigma^{2}-\alpha \sigma\right)\|u\|^{2}-\sigma(1-\sigma)\|\nabla u\|^{2}-c_{2} \sigma \int_{\mathbb{R}^{n}} F(x, u) \mathrm{d} x \\
& \quad+c\left(1+\left\|z\left(\theta_{t} \omega\right)\right\|^{2}+\left\|\nabla z\left(\theta_{t} \omega\right)\right\|^{2}+\left\|z\left(\theta_{t} \omega\right)\right\|_{H^{1}}^{k+1}\right)+\frac{2}{\alpha-\delta}\|g\|^{2} .
\end{aligned}
$$


Recalling the new norm $\|\cdot\|_{E}$ in (15), by (14) we obtain from (25) that

$$
\begin{aligned}
\frac{\mathrm{d}}{\mathrm{d} t}\left(\|\varphi\|_{E}^{2}+2 \int_{\mathbb{R}^{n}} F(x, u) \mathrm{d} x\right) \leqslant & -\delta\left(\|\varphi\|_{E}^{2}+2 \int_{\mathbb{R}^{n}} F(x, u) \mathrm{d} x\right)+\frac{2}{\alpha-\delta}\|g\|^{2} \\
& +c\left(1+\left\|z\left(\theta_{t} \omega\right)\right\|^{2}+\left\|\nabla z\left(\theta_{t} \omega\right)\right\|^{2}+\left\|z\left(\theta_{t} \omega\right)\right\|_{H^{1}}^{k+1}\right) .
\end{aligned}
$$

Using the Gronwall lemma, we have

$$
\begin{aligned}
& \left\|\varphi\left(t, \omega, \varphi_{0}(\omega)\right)\right\|_{E}^{2}+2 \int_{\mathbb{R}^{n}} F\left(x, u\left(t, \omega, u_{0}(\omega)\right)\right) \mathrm{d} x \\
& \leqslant \mathrm{e}^{-\delta t}\left(\left\|\varphi_{0}(\omega)\right\|_{E}^{2}+2 \int_{\mathbb{R}^{n}} F\left(x, u_{0}(\omega)\right) \mathrm{d} x\right)+\frac{2}{\alpha-\delta} \int_{0}^{t} \mathrm{e}^{\delta(s-t)}\|g\|^{2} \mathrm{~d} s \\
& +c \int_{0}^{t} \mathrm{e}^{\delta(s-t)}\left(1+\left\|z\left(\theta_{t} \omega\right)\right\|^{2}+\left\|\nabla z\left(\theta_{t} \omega\right)\right\|^{2}+\left\|z\left(\theta_{t} \omega\right)\right\|_{H^{1}}^{k+1}\right) \mathrm{d} s .
\end{aligned}
$$

Substituting $\omega$ by $\theta_{-t} \omega$, then we have from (27) that

$$
\begin{aligned}
& \left\|\varphi\left(t, \theta_{-t} \omega, \varphi_{0}\left(\theta_{-t} \omega\right)\right)\right\|_{E}^{2}+2 \int_{\mathbb{R}^{n}} F\left(x, u\left(t, \theta_{-t} \omega, u_{0}\left(\theta_{-t} \omega\right)\right)\right) \mathrm{d} x \\
& \leqslant \mathrm{e}^{-\delta t}\left(\left\|\varphi_{0}\left(\theta_{-t} \omega\right)\right\|_{E}^{2}+2 \int_{\mathbb{R}^{n}} F\left(x, u_{0}\left(\theta_{-t} \omega\right)\right) \mathrm{d} x\right)+\frac{2}{\alpha-\delta} \int_{0}^{t} \mathrm{e}^{\delta(s-t)}\|g\|^{2} \mathrm{~d} s \\
& \quad+c \int_{0}^{t} \mathrm{e}^{\delta(s-t)}\left(1+\left\|z\left(\theta_{s-t} \omega\right)\right\|^{2}+\left\|\nabla z\left(\theta_{s-t} \omega\right)\right\|^{2}+\left\|z\left(\theta_{s-t} \omega\right)\right\|_{H^{1}}^{k+1}\right) \mathrm{d} s \\
& \leqslant \mathrm{e}^{-\delta t}\left(\left\|\varphi_{0}\left(\theta_{-t} \omega\right)\right\|_{E}^{2}+2 \int_{\mathbb{R}^{n}} F\left(x, u_{0}\left(\theta_{-t} \omega\right)\right) \mathrm{d} x\right)+\frac{2}{\alpha-\delta} \int_{0}^{t} \mathrm{e}^{\delta(s-t)}\|g\|^{2} \mathrm{~d} s \\
& \quad+c \int_{-t}^{0} \mathrm{e}^{\delta s}\left(1+\left\|z\left(\theta_{s} \omega\right)\right\|^{2}+\left\|\nabla z\left(\theta_{s} \omega\right)\right\|^{2}+\left\|z\left(\theta_{s} \omega\right)\right\|_{H^{1}}^{k+1}\right) \mathrm{d} s .
\end{aligned}
$$

By (5), we get

$$
\int_{\mathbb{R}^{n}} F\left(x, u_{0}\left(\theta_{-t} \omega\right)\right) \mathrm{d} x \leqslant c\left(1+\left\|u_{0}\left(\theta_{-t} \omega\right)\right\|^{2}+\left\|u_{0}\left(\theta_{-t} \omega\right)\right\|^{k+1}\right)
$$

By assumption, $\{B(\omega)\} \in \mathscr{Y}$ is tempered. Then, by (29), if $\varphi_{0}\left(\theta_{-t} \omega\right) \in B\left(\theta_{-t} \omega\right)$, we have

$$
\lim _{t \rightarrow+\infty} \mathrm{e}^{-\delta t}\left(\left\|\varphi_{0}\left(\theta_{-t} \omega\right)\right\|_{E}^{2}+2 \int_{\mathbb{R}^{n}} F\left(x, u_{0}\left(\theta_{-t} \omega\right)\right) \mathrm{d} x\right)=0
$$

Note that $z\left(\theta_{t} \omega\right)=\sum_{j=1}^{m} h_{j} z_{j}\left(\theta_{t} \omega_{j}\right)$ and $h_{j} \in H^{1}\left(\mathbb{R}^{n}\right)(1 \leqslant j \leqslant m)$. By (8) with $\epsilon=\frac{\delta}{2}$, we obtain

$$
\begin{aligned}
& c \int_{-t}^{0} \mathrm{e}^{\delta s}\left(1+\left\|z\left(\theta_{s} \omega\right)\right\|^{2}+\left\|\nabla z\left(\theta_{s} \omega\right)\right\|^{2}+\left\|z\left(\theta_{s} \omega\right)\right\|_{H^{1}}^{k+1}\right) \mathrm{d} s \\
& \leqslant c \int_{-t}^{0} \mathrm{e}^{\delta s}\left(1+\mathrm{e}^{\frac{\delta|s|}{2}} \gamma(\omega)\right) \mathrm{d} s \\
& \quad=c\left(\delta\left(1-\mathrm{e}^{-\delta t}\right)+\frac{\delta}{2}\left(1-\mathrm{e}^{-\frac{\delta}{2} t}\right) \gamma(\omega)\right) .
\end{aligned}
$$

By (F3), we have that

$$
-2 \int_{\mathbb{R}^{n}} F\left(x, u\left(t, \theta_{-t} \omega, u_{0}\left(\theta_{-t} \omega\right)\right)\right) \mathrm{d} x \leqslant 2 \int_{\mathbb{R}^{n}} \eta_{3}(x) \mathrm{d} x \leqslant 2 \int_{\mathbb{R}^{n}}\left|\eta_{3}(x)\right| \mathrm{d} x
$$

Combining (28), (30), (31) and (32), there is a $T_{B}(\omega)>0$ such that for all $t \geqslant T_{B}(\omega)$,

$$
\left\|\varphi\left(t, \theta_{-t} \omega, \varphi_{0}\left(\theta_{-t} \omega\right)\right)\right\|_{E}^{2} \leqslant \varrho^{2}(\omega)
$$


where $\varrho^{2}(\omega)=c \delta(1+\gamma(\omega))$ Since $\gamma(\omega)$ is tempered, then $\left\{A_{0}(\omega)\right\} \in \mathscr{V}$, and $\left\{A_{0}(\omega)\right\}$ is a random absorbing set for $\bar{S}_{\sigma}(t, \omega)$ in $\mathcal{Y}$. So, the proof is completed.

To prove asymptotic compactness of the random dynamical system $\bar{S}_{\sigma}(t, \omega)$, we first prove that the solutions were uniformly small outside a bounded domain and then decomposed the solutions in a bounded domain in terms of eigenfunctions of negative Laplacian as in [20].

Given $r \geqslant 1$, denote by $\mathbb{H}_{r}=\left\{x \in \mathbb{R}^{n}:|x|<r\right\}$ and $\mathbb{R}^{n} \backslash \mathbb{H}_{r}$ the complement of $\mathbb{H}_{r}$.

Choose a smooth function $\rho$, such that $0 \leqslant \rho(s) \leqslant 1$ for $s \in \mathbb{R}$, and

$$
\rho(s)= \begin{cases}0, & 0 \leqslant|s| \leqslant 1, \\ 1, & |s| \leqslant 2\end{cases}
$$

and there exist constants $c_{5}, c_{6}$, such that $\left|\rho^{\prime}(s)\right| \leqslant c_{5},\left|\rho^{\prime \prime}(s)\right| \leqslant c_{6}$ for $s \in \mathbb{R}$.

Lemma 2 Assume that (F1)-(F4), $g \in L^{2}\left(\mathbb{R}^{n}\right)$ and $h_{j} \in H^{1}\left(\mathbb{R}^{n}\right)(1 \leqslant j \leqslant m)$ hold. Let $\{B(\omega)\} \in \mathscr{O}$ and $\varphi_{0}(\omega) \in B(\omega)$. Then, for every $\epsilon>0$, there exist $\tilde{T}=\tilde{T}(B, \varepsilon, \omega)>0$ and $\tilde{R}=\tilde{R}(\varepsilon, \omega) \geqslant 1$, such that the solution $\varphi$ of (9)-(10) satisfies for $\mathbb{P}$-a.e. $\omega \in \Omega, \forall t \geqslant \tilde{T}, \quad r \geqslant \tilde{R}$,

$$
\left\|\varphi\left(t, \theta_{-t} \omega, \varphi_{0}\left(\theta_{-t} \omega\right)\right)\right\|_{E\left(\mathbb{R}^{n} \mathbb{H}_{r}\right)}^{2} \leqslant \varepsilon
$$

Proof. We first consider the random Equations (9)-(10). Then taking the inner product of the second equation of (9) with $\rho\left(\frac{|x|^{2}}{r^{2}}\right) v$ in $L^{2}\left(\mathbb{R}^{n}\right)$, we obtain

$$
\begin{aligned}
& \frac{1}{2} \frac{\mathrm{d}}{\mathrm{d} t} \int_{\mathbb{R}^{n}} \rho\left(\frac{|x|^{2}}{r^{2}}\right)|v|^{2} \mathrm{~d} x \\
& =(\sigma-\alpha) \int_{\mathbb{R}^{n}} \rho\left(\frac{|x|^{2}}{r^{2}}\right)|v|^{2} \mathrm{~d} x-\int_{\mathbb{R}^{n}} \rho\left(\frac{|x|^{2}}{r^{2}}\right)|\nabla v|^{2} \mathrm{~d} x-\left(\lambda+\sigma^{2}-\alpha \sigma\right) \int_{\mathbb{R}^{n}} \rho\left(\frac{|x|^{2}}{r^{2}}\right) u v \mathrm{~d} x \\
& \quad+(1-\sigma) \int_{\mathbb{R}^{n}}(\Delta u) \rho\left(\frac{|x|^{2}}{r^{2}}\right) \nu \mathrm{d} x-\int_{\mathbb{R}^{n}} f(x, u) \rho\left(\frac{|x|^{2}}{r^{2}}\right) \nu \mathrm{d} x+\int_{\mathbb{R}^{n}} g(x) \rho\left(\frac{|x|^{2}}{r^{2}}\right) v \mathrm{~d} x \\
& \quad+\int_{\mathbb{R}^{n}}\left(\Delta z\left(\theta_{t} \omega\right)\right) \rho\left(\frac{|x|^{2}}{r^{2}}\right) v \mathrm{~d} x+(1+\sigma-\alpha) \int_{\mathbb{R}^{n}} \rho\left(\frac{|x|^{2}}{r^{2}}\right) z\left(\theta_{t} \omega\right) v \mathrm{~d} x .
\end{aligned}
$$

Substituting $v$ in (18) into the third, fourth and fifth terms on the left-hand side of (36), we get that

$$
\begin{aligned}
\int_{\mathbb{R}^{n}} \rho\left(\frac{|x|^{2}}{r^{2}}\right) u v \mathrm{~d} x & =\int_{\mathbb{R}^{n}} \rho\left(\frac{|x|^{2}}{r^{2}}\right) u\left(\frac{\mathrm{d} u}{\mathrm{~d} t}+\sigma u-z\left(\theta_{t} \omega\right)\right) \mathrm{d} x \\
& =\int_{\mathbb{R}^{n}} \rho\left(\frac{|x|^{2}}{r^{2}}\right)\left(\frac{1}{2} \frac{\mathrm{d}}{\mathrm{d} t} u^{2}+\sigma u^{2}-z\left(\theta_{t} \omega\right) u\right) \mathrm{d} x \\
& \geqslant \frac{1}{2} \frac{\mathrm{d}}{\mathrm{d} t} \int_{\mathbb{R}^{n}} \rho\left(\frac{|x|^{2}}{r^{2}}\right)|u|^{2} \mathrm{~d} x+\sigma \int_{\mathbb{R}^{n}} \rho\left(\frac{|x|^{2}}{r^{2}}\right)|u|^{2} \mathrm{~d} x-\int_{\mathbb{R}^{n}} \rho\left(\frac{|x|^{2}}{r^{2}}\right)\left|z\left(\theta_{t} \omega\right)\right| \cdot|u| \mathrm{d} x \\
& \geqslant \frac{1}{2} \frac{\mathrm{d}}{\mathrm{d} t} \int_{\mathbb{R}^{n}} \rho\left(\frac{|x|^{2}}{r^{2}}\right)|u|^{2} \mathrm{~d} x+\frac{\sigma}{2} \int_{\mathbb{R}^{n}} \rho\left(\frac{|x|^{2}}{r^{2}}\right)|u|^{2} \mathrm{~d} x-\frac{1}{2 \sigma} \int_{\mathbb{R}^{n}} \rho\left(\frac{|x|^{2}}{r^{2}}\right)\left|z\left(\theta_{t} \omega\right)\right|^{2} \mathrm{~d} x,
\end{aligned}
$$


Z. J. Wang, S. F. Zhou

$$
\begin{aligned}
& \int_{\mathbb{R}^{n}}(\Delta u) \rho\left(\frac{|x|^{2}}{r^{2}}\right) v \mathrm{~d} x=-\int_{\mathbb{R}^{n}}(\nabla u) \nabla\left(\rho\left(\frac{|x|^{2}}{r^{2}}\right) v\right) \mathrm{d} x \\
& =-\int_{\mathbb{R}^{n}}(\nabla u)\left(\frac{2 x}{r^{2}} \rho^{\prime}\left(\frac{|x|^{2}}{r^{2}}\right) v+\rho\left(\frac{|x|^{2}}{r^{2}}\right) \nabla\left(\frac{\mathrm{d} u}{\mathrm{~d} t}+\sigma u-z\left(\theta_{t} \omega\right)\right)\right) \mathrm{d} x \\
& \leqslant \int_{r<x<\sqrt{2} r} \frac{2 c_{5} x}{r^{2}}|(\nabla u) v| \mathrm{d} x-\frac{1}{2} \frac{\mathrm{d}}{\mathrm{d} t} \int_{\mathbb{R}^{n}} \rho\left(\frac{|x|^{2}}{r^{2}}\right)|\nabla u|^{2} \mathrm{~d} x-\sigma \int_{\mathbb{R}^{n}} \rho\left(\frac{|x|^{2}}{r^{2}}\right)|\nabla u|^{2} \mathrm{~d} x+\int_{\mathbb{R}^{n}} \rho\left(\frac{|x|^{2}}{r^{2}}\right)|\nabla u| \cdot\left|\nabla z\left(\theta_{t} \omega\right)\right| \mathrm{d} x \\
& \leqslant \int_{\mathbb{R}^{n}} \frac{2 \sqrt{2} c_{5}}{r}|(\nabla u) v| \mathrm{d} x-\frac{1}{2} \frac{\mathrm{d}}{\mathrm{d} t} \int_{\mathbb{R}^{n}} \rho\left(\frac{|x|^{2}}{r^{2}}\right)|\nabla u|^{2} \mathrm{~d} x-\frac{\sigma}{2} \int_{\mathbb{R}^{n}} \rho\left(\frac{|x|^{2}}{r^{2}}\right)|\nabla u|^{2} \mathrm{~d} x+\frac{1}{2 \sigma} \int_{\mathbb{R}^{n}} \rho\left(\frac{|x|^{2}}{r^{2}}\right)\left|\nabla z\left(\theta_{t} \omega\right)\right|^{2} \mathrm{~d} x \\
& \leqslant \frac{\sqrt{2} c_{5}}{r}\left(\left\|\left.\nabla u\right|^{2}+\right\| v \|^{2}\right)-\frac{1}{2} \frac{\mathrm{d}}{\mathrm{d} t} \int_{\mathbb{R}^{n}} \rho\left(\frac{|x|^{2}}{r^{2}}\right)|\nabla u|^{2} \mathrm{~d} x-\frac{\sigma}{2} \int_{\mathbb{R}^{n}} \rho\left(\frac{|x|^{2}}{r^{2}}\right)|\nabla u|^{2} \mathrm{~d} x+\frac{1}{2 \sigma} \int_{\mathbb{R}^{n}} \rho\left(\frac{|x|^{2}}{r^{2}}\right)\left|\nabla z\left(\theta_{t} \omega\right)\right|^{2} \mathrm{~d} x,
\end{aligned}
$$

By using conditions (F1), (F2) and (F3), we find

$$
\begin{aligned}
& -\int_{\mathbb{R}^{n}} f(x, u) \rho\left(\frac{|x|^{2}}{r^{2}}\right) \nu \mathrm{d} x=-\int_{\mathbb{R}^{n}} f(x, u) \rho\left(\frac{|x|^{2}}{r^{2}}\right)\left(\frac{\mathrm{d} u}{\mathrm{~d} t}+\sigma u-z\left(\theta_{t} \omega\right)\right) \mathrm{d} x \\
& =-\frac{\mathrm{d}}{\mathrm{d} t} \int_{\mathbb{R}^{n}} \rho\left(\frac{|x|^{2}}{r^{2}}\right) F(x, u) \mathrm{d} x-\sigma \int_{\mathbb{R}^{n}} \rho\left(\frac{|x|^{2}}{r^{2}}\right) f(x, u) u \mathrm{~d} x+\int_{\mathbb{R}^{n}} \rho\left(\frac{|x|^{2}}{r^{2}}\right) f(x, u) z\left(\theta_{t} \omega\right) \mathrm{d} x \\
& \leqslant-\frac{\mathrm{d}}{\mathrm{d} t} \int_{\mathbb{R}^{n}} \rho\left(\frac{|x|^{2}}{r^{2}}\right) F(x, u) \mathrm{d} x-c_{2} \sigma \int_{\mathbb{R}^{\mathbb{R}}} \rho\left(\frac{|x|^{2}}{r^{2}}\right) F(x, u) \mathrm{d} x-\sigma \int_{\mathbb{R}^{n}} \rho\left(\frac{|x|^{2}}{r^{2}}\right) \eta_{2}(x) \mathrm{d} x \\
& +\int_{\mathbb{R}^{\mathbb{R}}} \rho\left(\frac{|x|^{2}}{r^{2}}\right)\left(c_{1}|u|^{k}+\eta_{1}(x)\right)\left|z\left(\theta_{t} \omega\right)\right| \mathrm{d} x \\
& \leqslant-\frac{\mathrm{d}}{\mathrm{d} t} \int_{\mathbb{R}^{n}} \rho\left(\frac{|x|^{2}}{r^{2}}\right) F(x, u) \mathrm{d} x-c_{2} \sigma \int_{\mathbb{R}^{n}} \rho\left(\frac{|x|^{2}}{r^{2}}\right) F(x, u) \mathrm{d} x-\sigma \int_{\mathbb{R}^{n}} \rho\left(\frac{|x|^{2}}{r^{2}}\right) \eta_{2}(x) \mathrm{d} x \\
& +\frac{1}{2} \int_{\mathbb{R}^{n}} \rho\left(\frac{|x|^{2}}{r^{2}}\right)\left|\eta_{1}(x)\right|^{2} \mathrm{~d} x+\frac{1}{2} \int_{\mathbb{R}^{n}} \rho\left(\frac{|x|^{2}}{r^{2}}\right)\left|z\left(\theta_{t} \omega\right)\right|^{2} \mathrm{~d} x \\
& +c_{1}\left(\int_{\mathbb{R}^{n}} \rho\left(\frac{|x|^{2}}{r^{2}}\right)|u|^{k+1} \mathrm{~d} x\right)^{\frac{k}{k+1}}\left(\int_{\mathbb{R}^{n}} \rho\left(\frac{|x|^{2}}{r^{2}}\right) \mid z\left(\theta_{t} \omega\right)^{k+1} \mathrm{~d} x\right)^{\frac{1}{k+1}} \\
& \leqslant-\frac{\mathrm{d}}{\mathrm{d} t} \int_{\mathbb{R}^{n}} \rho\left(\frac{|x|^{2}}{r^{2}}\right) F(x, u) \mathrm{d} x-\frac{\sigma c_{2}}{2} \int_{\mathbb{R}^{n}} \rho\left(\frac{|x|^{2}}{r^{2}}\right) F(x, u) \mathrm{d} x-\sigma \int_{\mathbb{R}^{n}} \rho\left(\frac{|x|^{2}}{r^{2}}\right) \eta_{2}(x) \mathrm{d} x \\
& +\frac{1}{2} \int_{\mathbb{R}^{n}} \rho\left(\frac{|x|^{2}}{r^{2}}\right)\left|\eta_{1}(x)\right|^{2} \mathrm{~d} x+\frac{1}{2} \int_{\mathbb{R}^{n}} \rho\left(\frac{|x|^{2}}{r^{2}}\right)\left|z\left(\theta_{t} \omega\right)\right|^{2} \mathrm{~d} x \\
& +\frac{\sigma c_{2}}{2} \int_{\mathbb{R}^{n}} \rho\left(\frac{|x|^{2}}{r^{2}}\right) \eta_{3}(x) \mathrm{d} x+c \int_{\mathbb{R}^{n}} \rho\left(\frac{|x|^{2}}{r^{2}}\right)\left|z\left(\theta_{t} \omega\right)\right|^{k+1} \mathrm{~d} x .
\end{aligned}
$$

By the Cauchy-Schwartz inequality and the Young inequality, we obtain

346 


$$
\begin{aligned}
& \int_{\mathbb{R}^{n}} g(x) \rho\left(\frac{|x|^{2}}{r^{2}}\right) v \mathrm{~d} x \leqslant \frac{1}{\alpha-\sigma} \int_{\mathbb{R}^{n}} \rho\left(\frac{|x|^{2}}{r^{2}}\right)|g(x)|^{2} \mathrm{~d} x+\frac{\alpha-\sigma}{4} \int_{\mathbb{R}^{n}} \rho\left(\frac{|x|^{2}}{r^{2}}\right)|v|^{2} \mathrm{~d} x \\
& \int_{\mathbb{R}^{n}}\left(\Delta z\left(\theta_{t} \omega\right)\right) \rho\left(\frac{|x|^{2}}{r^{2}}\right) v \mathrm{~d} x=-\int_{\mathbb{R}^{n}}\left(\nabla z\left(\theta_{t} \omega\right)\right)\left(\frac{2 x}{r^{2}} \rho^{\prime}\left(\frac{|x|^{2}}{r^{2}}\right) v+\rho\left(\frac{|x|^{2}}{r^{2}}\right) \nabla v\right) \mathrm{d} x \\
& \leqslant \int_{r<x<\sqrt{2} r} \frac{2 c_{5} x}{r^{2}}\left|v \cdot \nabla z\left(\theta_{t} \omega\right)\right| \mathrm{d} x+\int_{\mathbb{R}^{n}} \rho\left(\frac{|x|^{2}}{r^{2}}\right)\left|\nabla v \cdot \nabla z\left(\theta_{t} \omega\right)\right| \mathrm{d} x \\
& \leqslant \int_{\mathbb{R}^{n}} \frac{2 \sqrt{2} c_{5}}{r}\left|v \cdot \nabla z\left(\theta_{t} \omega\right)\right| \mathrm{d} x+\int_{\mathbb{R}^{n}} \rho\left(\frac{|x|^{2}}{r^{2}}\right)|\nabla v|^{2} \mathrm{~d} x+\frac{1}{4} \int_{\mathbb{R}^{n}} \rho\left(\frac{|x|^{2}}{r^{2}}\right)\left|\nabla z\left(\theta_{t} \omega\right)\right|^{2} \mathrm{~d} x \\
& \leqslant \frac{\sqrt{2} c_{5}}{r}\left(\left\|\nabla z\left(\theta_{t} \omega\right)\right\|^{2}+\|v\|^{2}\right)+\int_{\mathbb{R}^{n}} \rho\left(\frac{|x|^{2}}{r^{2}}\right)|\nabla v|^{2} \mathrm{~d} x+\frac{1}{4} \int_{\mathbb{R}^{n}} \rho\left(\frac{|x|^{2}}{r^{2}}\right)\left|\nabla z\left(\theta_{t} \omega\right)\right|^{2} \mathrm{~d} x,
\end{aligned}
$$

$(1+\sigma-\alpha) \int_{\mathbb{R}^{n}} \rho\left(\frac{|x|^{2}}{r^{2}}\right) z\left(\theta_{t} \omega\right) v \mathrm{~d} x \leqslant\left(\alpha-\sigma-2+\frac{1}{\alpha-\sigma}\right) \int_{\mathbb{R}^{n}} \rho\left(\frac{|x|^{2}}{r^{2}}\right)\left|z\left(\theta_{t} \omega\right)\right|^{2} \mathrm{~d} x+\frac{\alpha-\sigma}{4} \int_{\mathbb{R}^{n}} \rho\left(\frac{|x|^{2}}{r^{2}}\right)|v|^{2} \mathrm{~d} x$

Then it follows from (37)-(42) that

$$
\begin{aligned}
& \frac{\mathrm{d}}{\mathrm{d} t} \int_{\mathbb{R}^{n}} \rho\left(\frac{|x|^{2}}{r^{2}}\right)\left(|v|^{2}+\left(\lambda+\sigma^{2}-\alpha \sigma\right)|u|^{2}+(1-\sigma)|\nabla u|^{2}+2 F(x, u)\right) \mathrm{d} x \\
& \leqslant \int_{\mathbb{R}^{n}} \rho\left(\frac{|x|^{2}}{r^{2}}\right)\left((\sigma-\alpha)|v|^{2}-\sigma\left(\lambda+\sigma^{2}-\alpha \sigma\right)|u|^{2}-\sigma(1-\sigma)|\nabla u|^{2}-c_{2} \sigma F(x, u)\right) \mathrm{d} x \\
& \quad+c \int_{\mathbb{R}^{n}} \rho\left(\frac{|x|^{2}}{r^{2}}\right)\left(\left|\eta_{1}(x)\right|^{2}+\left|\eta_{2}(x)\right|+\left|\eta_{3}(x)\right|+|g(x)|^{2}\right) \mathrm{d} x \\
& \quad+c \int_{\mathbb{R}^{n}} \rho\left(\frac{|x|^{2}}{r^{2}}\right)\left(\left|z\left(\theta_{t} \omega\right)\right|^{2}+\left|\nabla z\left(\theta_{t} \omega\right)\right|^{2}+\left|z\left(\theta_{t} \omega\right)\right|^{k+1}\right) \mathrm{d} x+\frac{c}{r}\left(\|\nabla u\|^{2}+\|v\|^{2}+\left\|\nabla z\left(\theta_{t} \omega\right)\right\|^{2}\right) .
\end{aligned}
$$

Letting

$$
X\left(t, \omega, X_{0}(\omega)\right)=\left|v\left(t, \omega, v_{0}(\omega)\right)\right|^{2}+\left(\lambda+\sigma^{2}-\alpha \sigma\right)\left|u\left(t, \omega, u_{0}(\omega)\right)\right|^{2}+(1-\sigma)\left|\nabla u\left(t, \omega, u_{0}(\omega)\right)\right|^{2}
$$

then, by (14) we have from (43) that

$$
\begin{aligned}
& \frac{\mathrm{d}}{\mathrm{d} t} \int_{\mathbb{R}^{n}} \rho\left(\frac{|x|^{2}}{r^{2}}\right)\left(X\left(t, \omega, X_{0}(\omega)\right)+2 F\left(x, u\left(t, \omega, u_{0}(\omega)\right)\right)\right) \mathrm{d} x \\
& \leqslant-\delta \int_{\mathbb{R}^{n}} \rho\left(\frac{|x|^{2}}{r^{2}}\right)\left(X\left(t, \omega, X_{0}(\omega)\right)+2 F\left(x, u\left(t, \omega, u_{0}(\omega)\right)\right)\right) \mathrm{d} x \\
& \quad+c \int_{\mathbb{R}^{n}} \rho\left(\frac{|x|^{2}}{r^{2}}\right)\left(\left|\eta_{1}(x)\right|^{2}+\left|\eta_{2}(x)\right|+\left|\eta_{3}(x)\right|+|g(x)|^{2}\right) \mathrm{d} x \\
& \quad+c \int_{\mathbb{R}^{n}} \rho\left(\frac{|x|^{2}}{r^{2}}\right)\left(\left|z\left(\theta_{t} \omega\right)\right|^{2}+\left|\nabla z\left(\theta_{t} \omega\right)\right|^{2}+\left|z\left(\theta_{t} \omega\right)\right|^{k+1}\right) \mathrm{d} x+\frac{c}{r}\left(\|\nabla u\|^{2}+\|v\|^{2}+\left\|\nabla z\left(\theta_{t} \omega\right)\right\|^{2}\right) .
\end{aligned}
$$

By using the Gronwall lemma, we get that 


$$
\begin{aligned}
& \int_{\mathbb{R}^{n}} \rho\left(\frac{|x|^{2}}{r^{2}}\right)\left(X\left(t, \omega, X_{0}(\omega)\right)+2 F\left(x, u\left(t, \omega, u_{0}(\omega)\right)\right)\right) \mathrm{d} x \\
& \leqslant \mathrm{e}^{-\delta t} \int_{\mathbb{R}^{n}} \rho\left(\frac{|x|^{2}}{r^{2}}\right)\left(X_{0}(\omega)+2 F\left(x, u_{0}(\omega)\right)\right) \mathrm{d} x \\
& \quad+c \int_{0}^{t} \mathrm{e}^{\delta(s-t)} \int_{\mathbb{R}^{n}} \rho\left(\frac{|x|^{2}}{r^{2}}\right)\left(\left|\eta_{1}(x)\right|^{2}+\left|\eta_{2}(x)\right|+\left|\eta_{3}(x)\right|+|g(x)|^{2}\right) \mathrm{d} x \mathrm{~d} s \\
& \quad+c \int_{0}^{t} \mathrm{e}^{\delta(s-t)} \int_{\mathbb{R}^{n}} \rho\left(\frac{|x|^{2}}{r^{2}}\right)\left(\left|z\left(\theta_{s} \omega\right)\right|^{2}+\left|\nabla z\left(\theta_{s} \omega\right)\right|^{2}+\left|z\left(\theta_{s} \omega\right)\right|^{k+1}\right) \mathrm{d} x \mathrm{~d} s \\
& \quad+\frac{c}{r} \int_{0}^{t} \mathrm{e}^{\delta(s-t)}\left(\left\|\nabla u\left(s, \omega, u_{0}(\omega)\right)\right\|^{2}+\left\|v\left(s, \omega, v_{0}(\omega)\right)\right\|^{2}+\left\|\nabla z\left(\theta_{s} \omega\right)\right\|^{2}\right) \mathrm{d} s .
\end{aligned}
$$

By replacing $\omega$ by $\theta_{-t} \omega$, it then follows from (46) that

$$
\begin{aligned}
& \int_{\mathbb{R}^{n}} \rho\left(\frac{|x|^{2}}{r^{2}}\right)\left(X\left(t, \theta_{-t} \omega, X_{0}\left(\theta_{-t} \omega\right)\right)+2 F\left(x, u\left(t, \theta_{-t} \omega, u_{0}\left(\theta_{-t} \omega\right)\right)\right)\right) \mathrm{d} x \\
& \leqslant \mathrm{e}^{-\delta t} \int_{\mathbb{R}^{n}} \rho\left(\frac{|x|^{2}}{r^{2}}\right)\left(X_{0}\left(\theta_{-t} \omega\right)+2 F\left(x, u_{0}\left(\theta_{-t} \omega\right)\right)\right) \mathrm{d} x \\
& \quad+c \int_{0}^{t} \mathrm{e}^{\delta(s-t)} \int_{\mathbb{R}^{n}} \rho\left(\frac{|x|^{2}}{r^{2}}\right)\left(\left|\eta_{1}(x)\right|^{2}+\left|\eta_{2}(x)\right|+\left|\eta_{3}(x)\right|+|g(x)|^{2}\right) \mathrm{d} x \mathrm{~d} s \\
& \quad+c \int_{-t}^{0} \mathrm{e}^{\delta s} \int_{\mathbb{R}^{n}} \rho\left(\frac{|x|^{2}}{r^{2}}\right)\left(\left|z\left(\theta_{s} \omega\right)\right|^{2}+\left|\nabla z\left(\theta_{s} \omega\right)\right|^{2}+\left|z\left(\theta_{s} \omega\right)\right|^{k+1}\right) \mathrm{d} x \mathrm{~d} s \\
& \quad+\frac{c}{r} \int_{0}^{t} \mathrm{e}^{\delta(s-t)}\left(\left\|\left.\nabla u\left(s, \theta_{-t} \omega, u_{0}\left(\theta_{-t} \omega\right)\right)\right|^{2}+\right\| v\left(s, \theta_{-t} \omega, v_{0}\left(\theta_{-t} \omega\right)\right)\left\|^{2}+\right\| \nabla z\left(\theta_{s-t} \omega\right) \|^{2}\right) \mathrm{d} s .
\end{aligned}
$$

By using (F3), there exists $\tilde{R}_{1}=\tilde{R}_{1}(\varepsilon, \omega)>0$, such that for all $r \geqslant \tilde{R}_{1}$,

$$
-\int_{\mathbb{R}^{n}} \rho\left(\frac{|x|^{2}}{r^{2}}\right) 2 F\left(x, u\left(t, \theta_{-t} \omega, u_{0}\left(\theta_{-t} \omega\right)\right)\right) \mathrm{d} x \leqslant 2 \int_{\mathbb{R}^{n}} \rho\left(\frac{|x|^{2}}{r^{2}}\right)\left|\eta_{3}(x)\right| \mathrm{d} x \leqslant \varepsilon
$$

In what follows, we estimate the terms on the right-hand side of (47). By (5), $\varphi_{0}\left(\theta_{-t} \omega\right) \in B\left(\theta_{-t} \omega\right)$ and the fact that $\{B(\omega)\}$ is tempered, we have that, there exists $\tilde{T}_{1}=\tilde{T}_{1}(B, \varepsilon, \omega)>0$, such that for all $t \geqslant \tilde{T}_{1}$,

$$
\begin{aligned}
& \mathrm{e}^{-\delta t} \int_{\mathbb{R}^{n}} \rho\left(\frac{|x|^{2}}{r^{2}}\right)\left(X_{0}\left(\theta_{-t} \omega\right)+2 F\left(x, u_{0}\left(\theta_{-t} \omega\right)\right)\right) \mathrm{d} x \leqslant \mathrm{e}^{-\delta t}\left(\left\|\varphi_{0}\left(\theta_{-t} \omega\right)\right\|_{E}^{2}+2 F\left(x, u_{0}\left(\theta_{-t} \omega\right)\right)\right) \\
& \leqslant \mathrm{e}^{-\delta t}\left(\left\|\varphi_{0}\left(\theta_{-t} \omega\right)\right\|_{E}^{2}+1+\left\|u_{0}\left(\theta_{-t} \omega\right)\right\|^{2}+\left\|u_{0}\left(\theta_{-t} \omega\right)\right\|^{k+1}\right) \leqslant \varepsilon .
\end{aligned}
$$

Since $g \in L^{2}\left(\mathbb{R}^{n}\right), \quad \eta_{1}(x) \in L^{2}(\mathbb{R}), \quad \eta_{2}(x) \in L^{1}(\mathbb{R})$ and $\eta_{3}(x) \in L^{1}(\mathbb{R})$, then, there is $\tilde{R}_{2}=\tilde{R}_{2}(\varepsilon, \omega)>1$, such that for $r \geqslant \tilde{R}_{2}$, the second term on the right-hand side of (47) satisfies

$$
\begin{gathered}
c \int_{0}^{t} \mathrm{e}^{\delta(s-t)} \int_{\mathbb{R}^{n}} \rho\left(\frac{|x|^{2}}{r^{2}}\right)\left(\left|\eta_{1}(x)\right|^{2}+\left|\eta_{2}(x)\right|+\left|\eta_{3}(x)\right|+|g(x)|^{2}\right) \mathrm{d} x \mathrm{~d} s \\
\leqslant \frac{c}{\delta} \int_{|x| \geqslant r} \rho\left(\frac{|x|^{2}}{r^{2}}\right)\left(\left|\eta_{1}(x)\right|^{2}+\left|\eta_{2}(x)\right|+\left|\eta_{3}(x)\right|+|g(x)|^{2}\right) \mathrm{d} x \leqslant \varepsilon .
\end{gathered}
$$


Note that $\left|z_{j}\left(\theta_{t} \omega_{j}\right)\right|$ is tempered, $z\left(\theta_{t} \omega\right)=\sum_{j=1}^{m} h_{j} z_{j}\left(\theta_{t} \omega_{j}\right)$ and $h_{j} \in H^{1}\left(\mathbb{R}^{n}\right)(1 \leqslant j \leqslant m)$. By (8) with $\epsilon=\frac{\delta}{2}$, there is $\tilde{R}_{3}=\tilde{R}_{3}(\varepsilon, \omega)>1$, such that for all $r \geqslant \tilde{R}_{3}$, the third term on the right-hand side of (47) satisfies

$$
\begin{aligned}
& c \int_{-t}^{0} \mathrm{e}^{\delta s} \int_{\mathbb{R}^{n}} \rho\left(\frac{|x|^{2}}{r^{2}}\right)\left(\left|z\left(\theta_{s} \omega\right)\right|^{2}+\left|\nabla z\left(\theta_{s} \omega\right)\right|^{2}+\left|z\left(\theta_{s} \omega\right)\right|^{k+1}\right) \mathrm{d} x \mathrm{~d} s \\
& \leqslant c \int_{-t}^{0} \mathrm{e}^{\delta s} \sum_{j=1}^{m} \int_{\mathbb{R}^{n}} \rho\left(\frac{|x|^{2}}{r^{2}}\right)\left(\left(\left|h_{j}\right|^{2}+\left|\nabla h_{j}\right|^{2}\right)\left|z_{j}\left(\theta_{s} \omega_{j}\right)\right|^{2}+\left|h_{j}\right|^{k+1}\left|z_{j}\left(\theta_{s} \omega_{j}\right)\right|^{k+1}\right) \mathrm{d} x \mathrm{~d} s \\
& \leqslant c \int_{-t}^{0} \mathrm{e}^{\delta s} \sum_{j=1}^{m}\left(\left|z_{j}\left(\theta_{s} \omega_{j}\right)\right|^{2}+\left|z_{j}\left(\theta_{s} \omega_{j}\right)\right|^{k+1}\right) \mathrm{d} s \sum_{j=1}^{m} \int_{\mathbb{R}^{n}} \rho\left(\frac{|x|^{2}}{r^{2}}\right)\left(\left|h_{j}\right|^{2}+\left|\nabla h_{j}\right|^{2}+\left|h_{j}\right|^{k+1}\right) \mathrm{d} x \\
& \leqslant c \int_{-t}^{0} \mathrm{e}^{\frac{\delta}{2} s} \gamma(\omega) \mathrm{d} s \sum_{j=1}^{m} \int_{\mathbb{R}^{n}} \rho\left(\frac{|x|^{2}}{r^{2}}\right)\left(\left|h_{j}\right|^{2}+\left|\nabla h_{j}\right|^{2}+\left|h_{j}\right|^{k+1}\right) \mathrm{d} x \\
& \leqslant \frac{2 c}{\delta} \gamma(\omega) \sum_{j=1}^{m} \int_{|x|>r}\left(\left|h_{j}\right|^{2}+\left|\nabla h_{j}\right|^{2}+\left|h_{j}\right|^{k+1}\right) \mathrm{d} x \\
& \leqslant \varepsilon
\end{aligned}
$$

Next, we estimate the forth term on the right-hand side of (47). Using (F3), replacing $t$ by $s$ and then $\omega$ by $\theta_{-t} \omega$ in (27), we have

$$
\begin{aligned}
\left\|\varphi\left(s, \theta_{-t} \omega, \varphi_{0}\left(\theta_{-t} \omega\right)\right)\right\|_{E}^{2} \leqslant & 2 \int_{\mathbb{R}^{n}}\left|\eta_{3}(x)\right| \mathrm{d} x+\mathrm{e}^{-\delta s}\left(\left\|\varphi_{0}\left(\theta_{-t} \omega\right)\right\|_{E}^{2}+2 \int_{\mathbb{R}^{n}} F\left(x, u_{0}\left(\theta_{-t} \omega\right)\right) \mathrm{d} x\right) \\
& +c \int_{0}^{s} \mathrm{e}^{\delta(\tau-s)}\left(1+\left\|z\left(\theta_{\tau-t} \omega\right)\right\|^{2}+\left\|\nabla z\left(\theta_{\tau-t} \omega\right)\right\|^{2}+\left\|z\left(\theta_{\tau-t} \omega\right)\right\|_{H^{1}}^{k+1}\right) \mathrm{d} \tau,
\end{aligned}
$$

it then follows that

$$
\begin{aligned}
& \frac{c}{r} \int_{0}^{t} \mathrm{e}^{\delta(s-t)}\left(\left\|\nabla u\left(s, \theta_{-t} \omega, u_{0}\left(\theta_{-t} \omega\right)\right)\right\|^{2}+\left\|v\left(s, \theta_{-t} \omega, v_{0}\left(\theta_{-t} \omega\right)\right)\right\|^{2}+\left\|\nabla z\left(\theta_{s-t} \omega\right)\right\|^{2}\right) \mathrm{d} s \\
& \leqslant \frac{c}{r} \int_{0}^{t} \mathrm{e}^{\delta(s-t)}\left(\left\|\varphi\left(s, \theta_{-t} \omega, \varphi_{0}\left(\theta_{-t} \omega\right)\right)\right\|^{2}+\left\|\nabla z\left(\theta_{s-t} \omega\right)\right\|^{2}\right) \mathrm{d} s \\
& \leqslant \frac{c}{r} \int_{0}^{t} \mathrm{e}^{\delta(s-t)}\left(2 \int_{\mathbb{R}^{n}}\left|\eta_{3}(x)\right| \mathrm{d} x+\mathrm{e}^{-\delta s}\left(\left\|\varphi_{0}\left(\theta_{-t} \omega\right)\right\|_{E}^{2}+2 \int_{\mathbb{R}^{n}} F\left(x, u_{0}\left(\theta_{-t} \omega\right)\right) \mathrm{d} x\right)\right. \\
& \left.\quad+c \int_{0}^{s} \mathrm{e}^{\delta(s-t)}\left(1+\left\|z\left(\theta_{\tau-t} \omega\right)\right\|^{2}+\left\|\nabla z\left(\theta_{\tau-t} \omega\right)\right\|^{2}+\left\|z\left(\theta_{\tau-t} \omega\right)\right\|_{H^{1}}^{k+1}\right) \mathrm{d} \tau\right) \mathrm{d} s+\frac{c}{r} \int_{0}^{t} \mathrm{e}^{\delta(s-t)}\left\|\nabla z\left(\theta_{s-t} \omega\right)\right\|^{2} \mathrm{~d} s \\
& \leqslant \frac{c}{r} \int_{0}^{t} \mathrm{e}^{-\delta t}\left(\left\|\varphi_{0}\left(\theta_{-t} \omega\right)\right\|_{E}^{2}+\left\|u_{0}\left(\theta_{-t} \omega\right)\right\|^{2}+\left\|u_{0}\left(\theta_{-t} \omega\right)\right\|_{H^{1}}^{k+1}\right) \mathrm{d} s \\
& \quad+\frac{c}{r} \int_{0}^{t} \int_{0}^{s} \mathrm{e}^{\delta(s-t)}\left(\left\|z\left(\theta_{\tau-t} \omega\right)\right\|^{2}+\left\|\nabla z\left(\theta_{\tau-t} \omega\right)\right\|^{2}+\left\|z\left(\theta_{\tau-t} \omega\right)\right\|_{H^{1}}^{k+1}\right) \mathrm{d} \tau \mathrm{d} s+\frac{c}{r}(1+\gamma(\omega)) \\
& \leqslant \frac{c}{r} \int_{0}^{t} \mathrm{e}^{-\delta t}\left(\left\|\varphi_{0}\left(\theta_{-t} \omega\right)\right\|_{E}^{2}+\left\|u_{0}\left(\theta_{-t} \omega\right)\right\|^{2}+\left\|u_{0}\left(\theta_{-t} \omega\right)\right\|_{H^{1}}^{k+1}\right) \mathrm{d} s \\
& \quad+\frac{c}{r} \int_{0}^{t} \int_{0}^{s} \mathrm{e}^{\frac{\delta}{2}(\tau-t)} \sum_{j=1}^{m}\left(\left\|h_{j}\right\|^{2}+\left\|\nabla h_{j}\right\|^{2}+\left\|h_{j}\right\|^{k+1}\right) \gamma(\omega) \mathrm{d} \tau \mathrm{d} s+\frac{c}{r}(1+\gamma(\omega)) \\
& \leqslant \frac{c}{r} \int_{0}^{t} \mathrm{e}^{-\delta t}\left(\left\|\varphi_{0}\left(\theta_{-t} \omega\right)\right\|_{E}^{2}+\left\|u_{0}\left(\theta_{-t} \omega\right)\right\|^{2}+\left\|u_{0}\left(\theta_{-t} \omega\right)\right\|_{H^{1}}^{k+1}\right) \mathrm{d} s \\
& \quad+\frac{c}{r} \sum_{j=1}^{m}\left(\left\|h_{j}\right\|^{2}+\left\|\nabla h_{j}\right\|^{2}+\left\|h_{j}\right\|^{k+1}\right) \gamma(\omega)+\frac{c}{r}(1+\gamma(\omega)) .
\end{aligned}
$$


Since $\gamma(\underset{\tilde{T}}{\omega})$ and $\{B(\omega)\}$ are tempered and $h_{j} \in H^{1}\left(\mathbb{R}^{n}\right)(1 \leqslant j \leqslant m)$, then for any $\varphi_{0}\left(\theta_{-t} \omega\right) \in B\left(\theta_{-t} \omega\right)$, there exist $\tilde{T}_{2}=\tilde{T}_{2}(B, \varepsilon, \omega)>0$ and $\tilde{R}_{4}=\tilde{R}_{4}(\varepsilon, \omega) \geqslant 1$, such that for all $t \geqslant \tilde{T}_{2}$ and $r \geqslant \tilde{R}_{4}$, we obtain

$$
\frac{c}{r} \int_{0}^{t} \mathrm{e}^{\delta(s-t)}\left(\left\|\nabla u\left(s, \theta_{-t} \omega, u_{0}\left(\theta_{-t} \omega\right)\right)\right\|^{2}+\left\|v\left(s, \theta_{-t} \omega, v_{0}\left(\theta_{-t} \omega\right)\right)\right\|^{2}+\left\|\nabla z\left(\theta_{s-t} \omega\right)\right\|^{2}\right) \mathrm{d} s \leqslant \varepsilon
$$

Letting $\tilde{T}=\max _{\tilde{T}}\left\{\tilde{T}_{1}, \tilde{T}_{2}\right\}$ and $\tilde{R}=\max \left\{\tilde{R}_{1}, \tilde{R}_{2}, \tilde{R}_{3}, \tilde{R}_{4}\right\}$, then, combining (48), (49), (50), (51) and (54), we have for all $t>\tilde{T}$ and $r>\tilde{R}$,

$$
\int_{\mathbb{R}^{n}} \rho\left(\frac{|x|^{2}}{r^{2}}\right) X\left(t, \theta_{-t} \omega, X_{0}\left(\theta_{-t} \omega\right)\right) \mathrm{d} x \leqslant 5 \varepsilon
$$

which implies

$$
\left\|\varphi\left(t, \theta_{-t} \omega, \varphi_{0}\left(\theta_{-t} \omega\right)\right)\right\|_{E\left(\mathbb{R}^{n} \backslash \mathbb{H}_{r}\right)}^{2} \leqslant 5 \varepsilon
$$

Then we complete the proof.

Let $\hat{\rho}=1-\rho$ with $\rho$ given by (35) and denote by $\hat{\rho}_{r}=\hat{\rho}\left(\frac{|x|^{2}}{r^{2}}\right)$. Fix $r \geqslant 1$ and set

$$
\left\{\begin{array}{l}
\hat{u}\left(t, \omega, u_{0}(\omega)\right)=\hat{\rho}_{r} u\left(t, \omega, u_{0}(\omega)\right), \\
\hat{v}\left(t, \omega, v_{0}(\omega)\right)=\hat{\rho}_{r}\left(t, \omega, v_{0}(\omega)\right), \\
\hat{z}\left(\theta_{t} \omega\right)=\hat{\rho}_{r} z\left(\theta_{t} \omega\right) .
\end{array}\right.
$$

Multiplying (9) by $\hat{\rho}_{r}$ and using (57) we find that

$$
\left\{\begin{aligned}
\frac{\mathrm{d} \hat{u}}{\mathrm{~d} t}= & \hat{v}-\sigma \hat{u}+\hat{z}\left(\theta_{t} \omega\right), \\
\frac{\mathrm{d} \hat{v}}{\mathrm{~d} t}= & (\sigma-\alpha) \hat{v}+\Delta \hat{v}-v \Delta \hat{\rho}_{r}-2 \nabla \nu \nabla \hat{\rho}_{r}+(1-\sigma)\left(\Delta \hat{u}-u \Delta \hat{\rho}_{r}-2 \nabla u \nabla \hat{\rho}_{r}\right) \\
& -\left(\lambda+\sigma^{2}-\alpha \sigma\right) \hat{u}-f(x, u) \hat{\rho}_{r}+g(x) \hat{\rho}_{r}+\Delta \hat{z}\left(\theta_{t} \omega\right)+(1+\sigma-\alpha) \hat{z}\left(\theta_{t} \omega\right) .
\end{aligned}\right.
$$

Considering the eigenvalue problem

$$
-\Delta \hat{u}=\hat{u} \text { in } \mathbb{H}_{2 r} \text {, with } \hat{u}=0 \text { on } \partial \mathbb{H}_{2 r}
$$

The problem has a family of eigenfunctions $\left\{e_{i}\right\}_{i \in \mathbb{N}}$ with the eigenvalues $\left\{\lambda_{i}\right\}_{i \in \mathbb{N}}$ :

$$
\lambda_{1} \leqslant \lambda_{2} \leqslant \cdots \leqslant \lambda_{i} \leqslant \cdots, \quad \lambda_{i} \rightarrow+\infty(i \rightarrow+\infty)
$$

such that $\left\{e_{i}\right\}_{i \in \mathbb{N}}$ is an orthonormal basis of $L^{2}\left(\mathbb{H}_{2 r}\right)$. Given $n$, let $X_{n}=\operatorname{span}\left\{e_{1}, \cdots, e_{n}\right\}$ and $P_{n}: L^{2}\left(\mathbb{H}_{2 r}\right) \rightarrow X_{n}$ be the projection operator.

Lemma 3 Assume that (F1)-(F4), $g \in L^{2}\left(\mathbb{R}^{n}\right)$ and $h_{j} \in H^{1}\left(\mathbb{R}^{n}\right)(1 \leqslant j \leqslant m)$ hold. Let $\{B(\omega)\} \in \mathscr{V}$ and $\varphi_{0}(\omega) \in B(\omega)$. Then, for every $\varepsilon>0$, there exist $\hat{T}=\hat{T}(B, \varepsilon, \omega)>0, \hat{R}=\hat{R}(\varepsilon, \omega) \geq 1$ and $N=N(\varepsilon, \omega)>0$, such that the solution $\varphi$ of (9)-(10) satisfies for $\mathbb{P}$-a.e. $\omega \in \Omega, \forall t \geqslant \hat{T}, \quad r \geqslant \hat{R}$ and $n \geqslant N$,

$$
\left\|\left(I-P_{n}\right) \hat{\varphi}\left(t, \theta_{-t} \omega, \varphi_{0}\left(\theta_{-t} \omega\right)\right)\right\|_{E\left(\mathbb{H}_{2 r}\right)}^{2} \leqslant \varepsilon
$$

Proof. Let $\hat{u}_{n, 1}=P_{n} \hat{u}, \hat{u}_{n, 2}=\left(I-P_{n}\right) \hat{u}, \hat{v}_{n, 1}=P_{n} \hat{v}, \hat{v}_{n, 2}=\left(I-P_{n}\right) \hat{v}, \quad \hat{z}_{n, 1}\left(\theta_{t} \omega\right)=P_{n} \hat{z}\left(\theta_{t} \omega\right)$, $\hat{z}_{n, 2}\left(\theta_{t} \omega\right)=\left(I-P_{n}\right) \hat{z}\left(\theta_{t} \omega\right)$. Applying $I-P_{n}$ to the first equation of (58), we obtain

$$
\hat{v}_{n, 2}=\frac{\mathrm{d} \hat{u}_{n, 2}}{\mathrm{~d} t}+\sigma \hat{u}_{n, 2}-\hat{z}_{n, 2}\left(\theta_{t} \omega\right)
$$


Then applying $I-P_{n}$ to the second equation of (58) and taking the inner product of the resulting equation with $\hat{v}_{n, 2}$ in $L^{2}\left(\mathbb{H}_{2 r}\right)$, we have

$$
\begin{aligned}
\frac{1}{2} \frac{\mathrm{d}}{\mathrm{d} t}\left\|\hat{v}_{n, 2}\right\|^{2}= & (\sigma-\alpha)\left\|\hat{v}_{n, 2}\right\|^{2}-\left\|\nabla \hat{v}_{n, 2}\right\|^{2}+(1-\sigma)\left(\Delta \hat{u}, \hat{v}_{n, 2}\right)-\left(\lambda+\sigma^{2}-\alpha \sigma\right)\left(\hat{u}_{n, 2}, \hat{v}_{n, 2}\right) \\
& -\left(\left(I-P_{n}\right) f(x, u) \hat{\rho}_{r}, \hat{v}_{n, 2}\right)-\left(\left(I-P_{n}\right)\left(v \Delta \hat{\rho}_{r}+2 \nabla v \nabla \hat{\rho}_{r}\right), \hat{v}_{n, 2}\right) \\
& -(1-\sigma)\left(\left(I-P_{n}\right)\left(u \Delta \hat{\rho}_{r}+2 \nabla u \nabla \hat{\rho}_{r}\right), \hat{v}_{n, 2}\right)+\left(\left(I-P_{n}\right) g(x) \hat{\rho}_{r}, \hat{v}_{n, 2}\right) \\
& +\left(\Delta \hat{z}_{n, 2}\left(\theta_{t} \omega\right)+(1+\sigma-\alpha) \hat{z}_{n, 2}\left(\theta_{t} \omega\right), \hat{v}_{n, 2}\right) .
\end{aligned}
$$

Substituting $\hat{v}_{n, 2}$ in (61) into the the third, fourth and fifth terms on the left-hand side of (62), we have

$$
\begin{aligned}
\left(\hat{u}_{n, 2}, \hat{v}_{n, 2}\right) & =\left(\hat{u}_{n, 2}, \frac{\mathrm{d} \hat{u}_{n, 2}}{\mathrm{~d} t}+\sigma \hat{u}_{n, 2}-\hat{z}_{n, 2}\left(\theta_{t} \omega\right)\right) \\
& \geqslant \frac{1}{2} \frac{\mathrm{d}}{\mathrm{d} t}\left\|\hat{u}_{n, 2}\right\|^{2}+\frac{\sigma}{2}\left\|\hat{u}_{n, 2}\right\|^{2}-\frac{1}{2 \sigma}\left\|\hat{z}_{n, 2}\left(\theta_{t} \omega\right)\right\|^{2}, \\
\left(\Delta \hat{u}_{n, 2}, \hat{v}_{n, 2}\right) & =-\left(\nabla \hat{u}_{n, 2}, \nabla\left(\frac{\mathrm{d} \hat{u}_{n, 2}}{\mathrm{~d} t}+\sigma \hat{u}_{n, 2}-\hat{z}_{n, 2}\left(\theta_{t} \omega\right)\right)\right) \\
& \leqslant-\frac{1}{2} \frac{\mathrm{d}}{\mathrm{d} t}\left\|\nabla \hat{u}_{n, 2}\right\|^{2}-\frac{3 \sigma}{4}\left\|\nabla \hat{u}_{n, 2}\right\|^{2}+\frac{1}{\sigma}\left\|\nabla \hat{z}_{n, 2}\left(\theta_{t} \omega\right)\right\|^{2}, \\
-\left(\left(I-P_{n}\right) f(x, u) \hat{\rho}_{r}, \hat{v}_{n, 2}\right) \quad-\left(\left(I-P_{n}\right) f(x, u) \hat{\rho}_{r}, \frac{\mathrm{d} \hat{u}_{n, 2}}{\mathrm{~d} t}+\sigma \hat{u}_{n, 2}-\hat{z}_{n, 2}\left(\theta_{t} \omega\right)\right) & \\
=-\left(\left(I-P_{n}\right) f_{u}(x, u) u_{t} \hat{\rho}_{r}, \hat{u}_{n, 2}\right) & -\sigma\left(\left(I-P_{n}\right) f(x, u) \hat{\rho}_{r}, \hat{u}_{n, 2}\right)+\left(\left(I-P_{n}\right) f(x, u) \hat{\rho}_{r}, \hat{z}_{n, 2}\left(\theta_{t} \omega\right)\right) .
\end{aligned}
$$

Using conditions (F1) and (F4), we have

$$
\begin{aligned}
& -\left(\left(I-P_{n}\right) f_{u}(x, u) u_{t} \hat{\rho}_{r}, \hat{u}_{n, 2}\right) \\
& \leqslant c\|u\|_{L^{6}}^{k-1} \cdot\left\|u_{t}\right\| \cdot\left\|\hat{u}_{n, 2}\right\| \frac{6}{L^{4-k}}+c\left\|\eta_{4}\right\|_{L^{6}} \cdot\left\|u_{t}\right\| \cdot\left\|\hat{u}_{n, 2}\right\|_{L^{3}} \\
& \leqslant c\|u\|_{L^{6}}^{k-1} \cdot\left\|u_{t}\right\| \cdot\left\|\hat{u}_{n, 2}\right\|^{\frac{3-k}{2}} \cdot\left\|\hat{u}_{n, 2}\right\|_{L^{6}}^{\frac{k-1}{2}}+c\left\|\eta_{4}\right\|_{L^{6}} \cdot\left\|u_{t}\right\| \cdot\left\|\hat{u}_{n, 2}\right\|_{L^{6}}^{\frac{1}{2}} \cdot\left\|\hat{u}_{n, 2}\right\|^{\frac{1}{2}} \\
& \leqslant c \lambda_{n+1}^{\frac{k-3}{4}}\|u\|_{H^{1}}^{k-1} \cdot\left\|u_{t}\right\| \cdot\left\|\nabla \hat{u}_{n, 2}\right\|+c \lambda_{n+1}^{-\frac{1}{4}}\left\|\eta_{4}\right\|_{H^{1}} \cdot\left\|u_{t}\right\| \cdot\left\|\nabla \hat{u}_{n, 2}\right\| \\
& \leqslant \frac{\sigma(1-\sigma)}{8}\left\|\nabla \hat{u}_{n, 2}\right\|^{2}+\frac{2 c}{\sigma(1-\sigma)}\left(\lambda_{n+1}^{\frac{k-3}{2}}\|u\|_{H^{1}}^{2(k-1)} \cdot\left\|u_{t}\right\|^{2}+\lambda_{n+1}^{-\frac{1}{2}}\left\|\eta_{4}\right\|_{H^{1}}^{2} \cdot\left\|u_{t}\right\|^{2}\right), \\
& -\sigma\left(\left(I-P_{n}\right) f(x, u) \hat{\rho}_{r}, \hat{u}_{n, 2}\right) \leqslant \sigma\left(c_{1}\left\|u^{k}\right\| \cdot\left\|\hat{u}_{n, 2}\right\|+\left\|\eta_{1}\right\| \cdot\left\|\hat{u}_{n, 2}\right\|\right) \\
& \leqslant \sigma \lambda_{n+1}^{-\frac{1}{2}}\left\|\nabla \hat{u}_{n, 2}\right\|\left(c_{1}\|u\|_{H^{1}}^{k}+\left\|\eta_{1}\right\|\right) \\
& \leqslant \frac{\sigma(1-\sigma)}{8}\left\|\nabla \hat{u}_{n, 2}\right\|^{2}+\frac{2 \sigma}{1-\sigma} \lambda_{n+1}^{-1}\left(c_{1}\|u\|_{H^{1}}^{k}+\left\|\eta_{1}\right\|\right)^{2} \text {, } \\
& \left(\left(I-P_{n}\right) f(x, u) \hat{\rho}_{r}, \hat{z}_{n, 2}\left(\theta_{t} \omega\right)\right) \leqslant\left(c_{1}\left\|\left(I-P_{n}\right) u^{k} \hat{\rho}_{r}\right\|+\left\|\left(I-P_{n}\right) \eta_{1} \hat{\rho}_{r}\right\|\right)\left\|\hat{z}_{n, 2}\left(\theta_{t} \omega\right)\right\| \\
& \leqslant\left(c\left\|\hat{u}_{n, 2}\right\|_{H^{1}}^{k}+\left\|\left(I-P_{n}\right) \eta_{1} \hat{\rho}_{r}\right\|\right)\left\|\hat{z}_{n, 2}\left(\theta_{t} \omega\right)\right\|,
\end{aligned}
$$


Z. J. Wang, S. F. Zhou

it then follows that

$$
\begin{aligned}
-\left(\left(I-P_{n}\right) f(x, u) \hat{\rho}_{r}, \hat{v}_{n, 2}\right) \leqslant & \frac{\sigma(1-\sigma)}{4}\left\|\nabla \hat{u}_{n, 2}\right\|^{2}+\frac{2 c}{\sigma(1-\sigma)}\left(\lambda_{n+1}^{\frac{k-3}{2}}\|u\|_{H^{1}}^{2(k-1)} \cdot\|u\|_{t}^{2}+\lambda_{n+1}^{-\frac{1}{2}}\left\|\eta_{4}\right\|_{H^{1}}^{2} \cdot\|u\|_{t}^{2}\right) \\
& +\frac{2 \sigma}{1-\sigma} \lambda_{n+1}^{-1}\left(c_{1}\|u\|_{H^{1}}^{k}+\left\|\eta_{1}\right\|\right)^{2}+\left(c\left\|\hat{u}_{n, 2}\right\|_{H^{1}}^{k}+\left\|\left(I-P_{n}\right) \eta_{1} \hat{\rho}_{r}\right\|\right)\left\|\hat{z}_{n, 2}\left(\theta_{t} \omega\right)\right\| .
\end{aligned}
$$

By using the Cauchy-Schwartz inequality and the Young inequality, we have

$$
\begin{aligned}
& -(1-\sigma)\left(\left(I-P_{n}\right)\left(u \Delta \hat{\rho}_{r}+2 \nabla u \nabla \hat{\rho}_{r}\right), \hat{v}_{n, 2}\right) \\
& =-(1-\sigma)\left(\left(I-P_{n}\right)\left(\frac{4 x^{2}}{r^{4}} u \hat{\rho}_{r}^{\prime \prime}+\frac{2}{r^{2}} u \hat{\rho}_{r}^{\prime}+\frac{4 x}{r^{2}} \nabla u \cdot \hat{\rho}_{r}^{\prime}\right), \hat{v}_{n, 2}\right) \\
& \leqslant(1-\sigma)\left(\frac{8 c_{6}+2 c_{5}}{r^{2}}\left\|\left(I-P_{n}\right) u\right\|+\frac{4 \sqrt{2} c_{5}}{r}\left\|\left(I-P_{n}\right) \nabla u\right\|\left\|\hat{v}_{n, 2}\right\|\right. \\
& \leqslant \frac{4(1-\sigma)^{2}}{\alpha-\sigma}\left(\frac{\left(8 c_{6}+2 c_{5}\right)^{2}}{r^{4}}\left\|\left(I-P_{n}\right) u\right\|^{2}+\frac{32 c_{5}^{2}}{r^{2}}\left\|\left(I-P_{n}\right) \nabla u\right\|^{2}\right)+\frac{\alpha-\sigma}{8}\left\|\hat{v}_{n, 2}\right\|^{2}, \\
& -\left(\left(I-P_{n}\right)\left(v \Delta \hat{\rho}_{r}+2 \nabla v \nabla \hat{\rho}_{r}\right), \hat{v}_{n, 2}\right) \\
& \quad=-\left(\left(I-P_{n}\right)\left(\frac{4 x^{2}}{r^{4}} v \hat{\rho}_{r}^{\prime \prime}+\frac{2}{r^{2}} v \hat{\rho}_{r}^{\prime}+\frac{4 x}{r^{2}} \nabla v \cdot \hat{\rho}_{r}^{\prime}\right), \hat{v}_{n, 2}\right) \\
& \leqslant \frac{8 c_{6}+2 c_{5}}{r^{2}}\left\|\left(I-P_{n}\right) v\right\| \cdot\left\|\hat{v}_{n, 2}\right\|+\frac{4 \sqrt{2} c_{5}}{r}\left\|\left(I-P_{n}\right) v\right\| \cdot\left\|\nabla \hat{v}_{n, 2}\right\| \\
& \leqslant\left(\frac{2\left(8 c_{6}+2 c_{5}\right)^{2}}{r^{4}(\alpha-\sigma)}+\frac{32 c_{5}^{2}}{r^{2}}\right)\left\|\left(I-P_{n}\right) v\right\|^{2}+\frac{\alpha-\sigma}{8}\left\|\hat{v}_{n, 2}\right\|^{2}+\frac{1}{2}\left\|\nabla \hat{v}_{n, 2}\right\|^{2}, \\
& \left(\left(I-P_{n}\right) g(x) \hat{\rho}_{r}, \hat{v}_{n, 2}\right) \leqslant \frac{2}{\alpha-\sigma}\left\|\left(I-P_{n}\right) g(x) \hat{\rho}_{r}\right\|^{2}+\frac{\alpha-\sigma}{8}\left\|\hat{v}_{n, 2}\right\|^{2} \\
& \left(\Delta \hat{z}_{n, 2}\left(\theta_{t} \omega\right), \hat{v}_{n, 2}\right)+(1+\sigma-\alpha)\left(\hat{z}_{n, 2}\left(\theta_{t} \omega\right), \hat{v}_{n, 2}\right) \\
& \leqslant\left\|\nabla \hat{z}_{n, 2}\left(\theta_{t} \omega\right)\right\| \cdot\left\|\nabla \hat{v}_{n, 2}\right\|+(1+\alpha-\sigma)\left\|\hat{\hat{z}}_{n, 2}\left(\theta_{t} \omega\right)\right\| \cdot\left\|\hat{v}_{n, 2}\right\| \\
& \leqslant \frac{1}{2}\left(\left\|\nabla \hat{z}_{n, 2}\left(\theta_{t} \omega\right)\right\|^{2}+\left\|\nabla \hat{v}_{n, 2}\right\|^{2}\right)+\frac{\alpha-\sigma}{8}\left\|\hat{v}_{n, 2}\right\|^{2}+\frac{2(1+\alpha-\sigma)^{2}}{\alpha-\sigma}\left\|\hat{z}_{n, 2}\left(\theta_{t} \omega\right)\right\|^{2} .
\end{aligned}
$$

From (63)-(73) we can obtain that

$$
\begin{aligned}
& \frac{\mathrm{d}}{\mathrm{d} t}\left(\left\|\hat{v}_{n, 2}\right\|^{2}+\left(\lambda+\sigma^{2}-\alpha \sigma\right)\left\|\hat{u}_{n, 2}\right\|^{2}+(1-\sigma)\left\|\nabla \hat{u}_{n, 2}\right\|^{2}\right) \\
& \leqslant(\sigma-\alpha)\left\|\hat{v}_{n, 2}\right\|^{2}-\sigma\left(\lambda+\sigma^{2}-\alpha \sigma\right)\left\|\hat{u}_{n, 2}\right\|\left\|^{2}-(1-\sigma)\right\| \nabla \hat{u}_{n, 2} \|^{2} \\
& \quad+c\left(\left\|\hat{z}_{n, 2}\left(\theta_{t} \omega\right)\right\|+\left\|\hat{z}_{n, 2}\left(\theta_{t} \omega\right)\right\|^{2}+\left\|\nabla \hat{z}_{n, 2}\left(\theta_{t} \omega\right)\right\|^{2}\right)+c \lambda_{n+1}^{\frac{k-3}{2}}\|u\|_{H^{1}}^{2(k-1)} \cdot\left\|u_{t}\right\|^{2} \\
& \quad+c \lambda_{n+1}^{-\frac{1}{2}}\left\|\eta_{4}\right\|_{H^{1}}^{2} \cdot\left\|u_{t}\right\|^{2}+c \lambda_{n+1}^{-1}\left(\|u\|_{H^{1}}^{k}+\left\|\eta_{1}\right\|\right)^{2}+\frac{c}{r^{4}}\left\|\left(I-P_{n}\right) u\right\|^{2} \\
& \quad+\frac{c}{r^{2}}\left\|\left(I-P_{n}\right) \nabla u\right\|^{2}+\frac{c}{r^{2}}\left(\frac{1}{r^{2}}+1\right)\left\|\left(I-P_{n}\right) v\right\|^{2}+c\left\|\left(I-P_{n}\right) g(x) \hat{\rho}_{r}\right\|^{2} .
\end{aligned}
$$

352 
Since $\lambda_{n+1} \rightarrow \infty$ there exist $\hat{N}_{1}=\hat{N}_{1}(\varepsilon, \omega)>0$ and $\hat{R}=\hat{R}(\varepsilon, \omega)>1$, such that if $r>\hat{R}$ and $n>\hat{N}_{1}$, then by (14) and the new norm $\|\cdot\|_{E}$ in (15), we have

$$
\begin{aligned}
\frac{\mathrm{d}}{\mathrm{d} t}\left\|\hat{\varphi}_{n, 2}\right\|_{E\left(\mathbb{H}_{2 r}\right)}^{2} & \\
\leqslant & -\delta\left\|\hat{\varphi}_{n, 2}\right\|_{E\left(\mathbb{H}_{2 r}\right)}^{2}+c\left(\left\|\hat{z}_{n, 2}\left(\theta_{t} \omega\right)\right\|^{2}+\left\|\nabla \hat{z}_{n, 2}\left(\theta_{t} \omega\right)\right\|^{2}\right)+c \varepsilon\|u\|_{H^{1}}^{2(k-1)} \cdot\left\|u_{t}\right\|^{2}+c \varepsilon\left\|u_{t}\right\|^{2}+c \varepsilon\left(\|u\|_{H^{1}}^{k}+\left\|\eta_{1}\right\|\right)^{2} \\
& +c \varepsilon\left\|\left(I-P_{n}\right) u\right\|^{2}+c \varepsilon\left\|\left(I-P_{n}\right) \nabla u\right\|^{2}+c \varepsilon\left\|\left(I-P_{n}\right) v\right\|^{2}+c\left\|\left(I-P_{n}\right) g(x) \hat{\rho}_{r}\right\|^{2} \\
\leqslant & -\delta\left\|\hat{\varphi}_{n, 2}\right\|_{E\left(\mathbb{H}_{2 r}\right)}^{2}+c\left(\left\|\hat{z}_{n, 2}\left(\theta_{t} \omega\right)\right\|^{2}+\left\|\nabla \hat{z}_{n, 2}\left(\theta_{t} \omega\right)\right\|^{2}\right)+c \varepsilon\left(1+\|u\|_{H^{1}}^{2(k-1)} \cdot\left\|u_{t}\right\|^{2}+\left\|u_{t}\right\|^{2}+\|u\|_{H^{1}}^{2 k}+\left\|\left(I-P_{n}\right) \varphi\right\|_{E}^{2}\right) \\
\leqslant & -\delta\left\|\hat{\varphi}_{n, 2}\right\|_{E\left(\mathbb{H}_{2 r}\right)}^{2}+c\left(\left\|\hat{z}_{n, 2}\left(\theta_{t} \omega\right)\right\|^{2}+\left\|\nabla \hat{z}_{n, 2}\left(\theta_{t} \omega\right)\right\|^{2}\right)+c \varepsilon\left(1+\left\|u_{t}\right\|^{6}+\|u\|_{H^{1}}^{6}+\left\|\left(I-P_{n}\right) \varphi\right\|_{E}^{2}\right) .
\end{aligned}
$$

Using the Gronwall lemma, we have

$$
\begin{aligned}
& \left\|\hat{\varphi}_{n, 2}\left(t, \omega, \varphi_{n, 2,0}(\omega)\right)\right\|_{E\left(\mathbb{H}_{2 r}\right)}^{2} \\
& \quad \leqslant \mathrm{e}^{-\delta t}\left\|\varphi_{n, 2,0}(\omega)\right\|_{E\left(\mathbb{H}_{2 r}\right)}^{2}+c \int_{0}^{t} \mathrm{e}^{\delta(s-t)}\left(\left\|\hat{z}_{n, 2}\left(\theta_{s} \omega\right)\right\|^{2}+\left\|\nabla \hat{z}_{n, 2}\left(\theta_{s} \omega\right)\right\|^{2}\right) \mathrm{d} s \\
& \quad+c \varepsilon \int_{0}^{t} \mathrm{e}^{\delta(s-t)}\left(1+\left\|u_{t}\left(s, \omega, u_{0}(\omega)\right)\right\|^{6}+\left\|u\left(s, \omega, u_{0}(\omega)\right)\right\|_{H^{1}}^{6}+\left\|\left(I-P_{n}\right) \varphi\left(s, \omega, \varphi_{0}(\omega)\right)\right\|_{E}^{2}\right) \mathrm{d} s .
\end{aligned}
$$

By substituting $\omega$ by $\theta_{-t} \omega$, we can get from (76) that,

$$
\begin{aligned}
& \left\|\hat{\varphi}_{n, 2}\left(t, \theta_{-t} \omega, \varphi_{n, 2,0}\left(\theta_{-t} \omega\right)\right)\right\|_{E\left(\mathbb{H}_{2 r}\right)}^{2} \leqslant \mathrm{e}^{-\delta t}\left\|\hat{\varphi}_{n, 2,0}\left(\theta_{-t} \omega\right)\right\|_{E\left(\mathbb{H}_{2 r}\right)}^{2}+c \int_{0}^{t} \mathrm{e}^{\delta(s-t)}\left(\left\|\hat{z}_{n, 2}\left(\theta_{s-t} \omega\right)\right\|^{2}+\left\|\nabla \hat{z}_{n, 2}\left(\theta_{s-t} \omega\right)\right\|^{2}\right) \mathrm{d} s \\
& +c \varepsilon \int_{0}^{t} \mathrm{e}^{\delta(s-t)}\left(1+\left\|u_{t}\left(s, \theta_{-t} \omega, u_{0}\left(\theta_{-t} \omega\right)\right)\right\|^{6}+\left\|u\left(s, \theta_{-t} \omega, u_{0}\left(\theta_{-t} \omega\right)\right)\right\|_{H^{1}}^{6}+\left\|\left(I-P_{n}\right) \varphi\left(s, \theta_{-t} \omega, \varphi_{0}\left(\theta_{-t} \omega\right)\right)\right\|_{E}^{2}\right) \mathrm{d} s .
\end{aligned}
$$

We next estimate each term on the right-hand side of (77). Since $\varphi_{0}\left(\theta_{-t} \omega\right) \in B\left(\theta_{-t} \omega\right)$ and the fact that $\{B(\omega)\}$ is tempered, there exist $\hat{T}_{1}=\hat{T}_{1}(B, \varepsilon, \omega)>0$ and $\hat{N}_{2}=\hat{N}_{2}(\varepsilon, \omega)>0$, such that if $t>\hat{T}_{1}$ and $n>\hat{N}_{2}$, then

$$
\mathrm{e}^{-\delta t}\left\|\hat{\varphi}_{n, 2,0}\left(\theta_{-t} \omega\right)\right\|_{E\left(\mathbb{H}_{2 r}\right)}^{2} \leqslant \varepsilon
$$

Since $\left|z_{j}\left(\theta_{t} \omega_{j}\right)\right|$ is tempered, $z\left(\theta_{t} \omega\right)=\sum_{j=1}^{m} h_{j} z_{j}\left(\theta_{t} \omega_{j}\right)$ and $h_{j} \in H^{1}\left(\mathbb{R}^{n}\right)(1 \leqslant j \leqslant m)$, then, by (8) with $\epsilon=\frac{\delta}{2}$ there are $\hat{N}_{3}=\hat{N}_{3}(\varepsilon, \omega)>0$ and $\hat{T}_{2}=\hat{T}_{2}(\varepsilon, \omega)>0$, such that for all $n>\hat{N}_{3}$ and $t>\hat{T}_{2}$, the second term on the right-hand side of (77) satisfies

$$
\begin{aligned}
c \int_{0}^{t} \mathrm{e}^{\delta(s-t)}\left(\left\|\hat{\mathrm{z}}_{n, 2}\left(\theta_{s-t} \omega\right)\right\|^{2}+\left\|\nabla \hat{z}_{n, 2}\left(\theta_{s-t} \omega\right)\right\|^{2}\right) \mathrm{d} s & \leqslant c \int_{-t}^{0} \mathrm{e}^{\delta s}\left(\left\|\hat{z}_{n, 2}\left(\theta_{s} \omega\right)\right\|^{2}+\left\|\nabla \hat{z}_{n, 2}\left(\theta_{s} \omega\right)\right\|^{2}\right) \mathrm{d} s \\
& \leqslant c \int_{-t}^{0} \mathrm{e}^{\delta s} \sum_{j=1}^{m} \int_{\mathbb{R}^{n}}\left(I-P_{n}\right) \hat{\rho}_{r}\left(\left|h_{j}\right|^{2}+\left|\nabla h_{j}\right|^{2}\right)\left|z_{j}\left(\theta_{s} \omega_{j}\right)\right|^{2} \mathrm{~d} x \mathrm{~d} s \\
& \leqslant c \varepsilon \int_{-t}^{0} \mathrm{e}^{\delta s} \sum_{j=1}^{m}\left|z_{j}\left(\theta_{s} \omega_{j}\right)\right|^{2} \mathrm{~d} s \\
& \leqslant \frac{2 c \varepsilon}{\delta} \gamma(\omega) \\
& \leqslant \varepsilon .
\end{aligned}
$$


Next, we estimate the third term on the right-hand side of (77). By (6), (18) and (33),

$$
\begin{aligned}
& c \varepsilon \int_{0}^{t} \mathrm{e}^{\delta(s-t)}\left(1+\left\|u_{t}\left(s, \theta_{-t} \omega, u_{0}\left(\theta_{-t} \omega\right)\right)\right\|^{6}+\left\|u\left(s, \theta_{-t} \omega, u_{0}\left(\theta_{-t} \omega\right)\right)\right\|_{H^{1}}^{6}+\left\|\left(I-P_{n}\right) \varphi\left(s, \theta_{-t} \omega, \varphi_{0}\left(\theta_{-t} \omega\right)\right)\right\|_{E}^{2}\right) \mathrm{d} s \\
& \leqslant c \varepsilon \int_{0}^{t} \mathrm{e}^{\delta(s-t)}\left(1+\left\|u\left(s, \theta_{-t} \omega, u_{0}\left(\theta_{-t} \omega\right)\right)\right\|^{6}+\left\|v\left(s, \theta_{-t} \omega, u_{0}\left(\theta_{-t} \omega\right)\right)\right\|^{6}+\|z(\omega)\|^{6}+\left\|u\left(s, \theta_{-t} \omega, u_{0}\left(\theta_{-t} \omega\right)\right)\right\|_{H^{1}}^{6}\right. \\
& \left.\quad+\left\|\varphi\left(s, \theta_{-t} \omega, \varphi_{0}\left(\theta_{-t} \omega\right)\right)\right\|_{E}^{2}\right) \mathrm{d} s \\
& \leqslant c \varepsilon \int_{0}^{t} \mathrm{e}^{\delta(s-t)}\left(1+\left\|\varphi\left(s, \theta_{-t} \omega, \varphi_{0}\left(\theta_{-t} \omega\right)\right)\right\|_{E}^{6}+\|z(\omega)\|^{6}+\left\|\varphi\left(s, \theta_{-t} \omega, \varphi_{0}\left(\theta_{-t} \omega\right)\right)\right\|_{E}^{2}\right) \mathrm{d} s \\
& \leqslant c \varepsilon \int_{0}^{t} \mathrm{e}^{\delta(s-t)}\left(1+\varrho^{2}(\omega)+\varrho^{6}(\omega)+\gamma^{6}(\omega)\right) \mathrm{d} s \\
& \leqslant c \varepsilon \delta\left(1+\varrho^{2}(\omega)+\varrho^{6}(\omega)+\gamma^{6}(\omega)\right),
\end{aligned}
$$

which implies that there exists $\hat{T}_{3}=\hat{T}_{3}(B, \varepsilon, \omega)>0$, such that for $t>\hat{T}_{3}$,

$$
c \varepsilon \int_{0}^{t} e^{\delta(s-t)}\left(1+\left\|u_{t}\left(s, \theta_{-t} \omega, u_{0}\left(\theta_{-t} \omega\right)\right)\right\|^{6}+\left\|u\left(s, \theta_{-t} \omega, u_{0}\left(\theta_{-t} \omega\right)\right)\right\|_{H^{1}}^{6}+\left\|\left(I-P_{n}\right) \varphi\left(s, \theta_{-t} \omega, \varphi_{0}\left(\theta_{-t} \omega\right)\right)\right\|_{E}^{2}\right) \mathrm{d} s \leqslant \varepsilon
$$

Let $N=\max \left\{N_{1}, N_{2}, N_{3}\right\}$ and $\hat{T}=\max \left\{\hat{T}_{1}, \hat{T}_{2}, \hat{T}_{3}\right\}$. Then, it follows from (78), (79) and (81) that, for all $t>\hat{T}, r>\hat{R}$ and $n \geqslant N$,

$$
\left\|\hat{\varphi}_{n, 2}\left(t, \theta_{-t} \omega, \varphi_{n, 2,0}\left(\theta_{-t} \omega\right)\right)\right\|_{E\left(\mathbb{H}_{2 r}\right)}^{2} \leqslant 3 \varepsilon
$$

which completes the proof.

\subsection{Random Attractor}

In this subsection, we prove the existence of a global random attractor for the random dynamical system generated by (9)-(10).

Theorem 2 Assume that (F1)-(F4), $g \in L^{2}\left(\mathbb{R}^{n}\right)$ and $h_{j} \in H^{1}\left(\mathbb{R}^{n}\right)(1 \leqslant j \leqslant m)$ hold. Let $\{B(\omega)\} \in \mathscr{Y}$ and $\varphi_{0}(\omega) \in B(\omega)$. Then the random dynamical system $\bar{S}_{\sigma}(t, \omega)$ generated by (9)-(10) has a unique global random attractor in $E$.

Proof. Notice that the random dynamical system $\bar{S}_{\sigma}(t, \omega)$ has a random absorbing set $\{A(\omega)\}$ in $\mathscr{O}$ by Lemma 1.

Next, we will prove that the random dynamical system $\bar{S}_{\sigma}(t, \omega)$ is asymptotically compact in $E$.

Let $t_{m} \rightarrow \infty,\{B(\omega)\} \in \mathscr{O}$, and $\varphi_{0}\left(\theta_{-t_{m}} \omega\right) \in B\left(\theta_{-t_{m}} \omega\right)$. Using Lemma 1 , we find that

$$
\left\{\varphi\left(t_{m}, \theta_{-t_{m}} \omega, \varphi_{0}\left(\theta_{-t_{m}} \omega\right)\right)\right\}
$$

is a bounded in $E$; that is, for $\mathbb{P}$-a.e. $\omega \in \Omega$, there exists $M_{1}=M_{1}(\varepsilon, \omega)>0$ such that for all $m>M_{1}$,

$$
\left\|\varphi\left(t_{m}, \theta_{-t_{m}} \omega, \varphi_{0}\left(\theta_{-t_{m}} \omega\right)\right)\right\|_{E}^{2} \leqslant \varrho^{2}(\omega)
$$

By Lemma 2, we have that there are $k_{1}=k_{1}(\varepsilon, \omega)>0$ and $\hat{M}_{2}=\hat{M}_{2}(B, \varepsilon, \omega)>0$, such that for every $m \geqslant \hat{M}_{2}$,

$$
\left\|\hat{\varphi}\left(t_{m}, \theta_{-t_{m}} \omega, \varphi_{0}\left(\theta_{-t_{m}} \omega\right)\right)\right\|_{E\left(\mathbb{R}^{n} \backslash \mathbb{H}_{k_{1}}\right)}^{2} \leqslant \varepsilon
$$

In addition, it follows from Lemma 3 that there exist $N=N(\varepsilon, \omega)>0, k_{2}=k_{2}(\varepsilon, \omega) \geqslant k_{1}$ and $\hat{M}_{3}=\hat{M}_{3}(B, \varepsilon, \omega)>0$, such that for every $m \geqslant \hat{M}_{3}$,

$$
\left\|\left(I-P_{N}\right) \hat{\varphi}\left(t_{m}, \theta_{-t_{m}} \omega, \varphi_{0}\left(\theta_{-t_{m}} \omega\right)\right)\right\|_{E\left(\mathbb{H}_{2 k_{2}}\right)}^{2} \leqslant \varepsilon
$$


Then, by (57) and (83), $\left\{P_{N} \hat{\varphi}\left(t_{m}, \theta_{-t_{m}} \omega, \varphi_{0}\left(\theta_{-t_{m}} \omega\right)\right)\right\}$ is a bounded in $P_{N} E\left(\mathbb{H}_{2 k_{2}}\right)$, which together with (85) implies that $\left\{\hat{\varphi}\left(t_{m}, \theta_{-t_{m}} \omega, \varphi_{0}\left(\theta_{-t_{m}} \omega\right)\right)\right\}$ is precompact in $H_{0}^{1}\left(\mathbb{H}_{2 k_{2}}\right) \times L^{2}\left(\mathbb{H}_{2 k_{2}}\right)$. Recalling (57), we find that $\left\{\varphi\left(t_{m}, \theta_{-t_{m}} \omega, \varphi_{0}\left(\theta_{-t_{m}} \omega\right)\right)\right\}$ is precompact in $E\left(\mathbb{H}_{k_{2}}\right)$, which along with (84) and (12) shows that the random dynamical system $\bar{S}_{\sigma}(t, \omega)$ is asymptotically compact in $E$.

Then, by Theorem 1 , the random dynamical system $\bar{S}_{\sigma}(t, \omega)$ generated by (9)-(10) has a unique global random attractor in $E$.

\section{Remarks}

In the present article, we have discussed the existence of a random attractor to the stochastic strongly damped wave equation with additive noise defined on unbounded domains. It is also interesting to consider the the same problem for stochastic strongly damped wave equation with multiplicative noise $\epsilon u \frac{\mathrm{d} W}{\mathrm{~d} t}$. In this case, the coefficient $\epsilon$ of the noise term needs to be suitable small, which is different from (1) that with additive white noise $\sum_{j=1}^{m} h_{j}(x) \frac{\mathrm{d} W_{j}}{\mathrm{~d} t}$, this is because that the multiplicative noise $\epsilon u \frac{\mathrm{d} W}{\mathrm{~d} t}$ depends on the state variable $u$, but the additive noise term $\sum_{j=1}^{m} h_{j}(x) \frac{\mathrm{d} W_{j}}{\mathrm{~d} t}$ is independent of $u$. The authors will pursue this line of research in the future.

\section{Acknowledgments}

We thank the editor and the referee for their comments. The authors are supported by National Natural Science Foundation of China (Nos. 11326114, 11401244, 11071165 and 11471290); Natural Science Research Project of Ordinary Universities in Jiangsu Province (No. 14KJB110003); Zhejiang Natural Science Foundation under Grant No. LY14A010012 and Zhejiang Normal University Foundation under Grant No. ZC304014012. This support is greatly appreciated.

\section{References}

[1] Arnold, L. (1998) Random Dynamical Systems. Springer-Verlag, New York and Berlin. http://dx.doi.org/10.1007/978-3-662-12878-7

[2] Belleri, V. and Pata, V. (2001) Attractors for Semilinear Strongly Damped Wave Equations on $\mathbb{R}^{3}$. Discrete and Continuous Dynamical Systems, 7, 719-735. http://dx.doi.org/10.3934/dcds.2001.7.719

[3] Conti, M., Pata, V. and Squassina, M. (2005) Strongly Damped Wave Equations on $\mathbb{R}^{3}$ with Critical Nonlinearities. Communications on Pure and Applied Analysis, 9, 161-176.

[4] Chen, F., Guo, B. and Wang, P. (1998) Long Time Behavior of Strongly Damped Nonlinear Wave Equations. Journal of Differential Equations, 147, 339-352. http://dx.doi.org/10.1006/jdeq.1998.3447

[5] Li, H. and Zhou, S. (2008) On Non-Autonomous Strongly Damped Wave Equations with a Uniform Attractor and Some Averaging. Journal of Mathematical Analysis and Applications, 341, 791-802. http://dx.doi.org/10.1016/j.jmaa.2007.10.051

[6] Temam, R. (1998) Infinite Dimensional Dynamical Systems in Mechanics and Physics. Springer-Verlag, New York.

[7] Yang, M. and Sun, C. (2009) Attractors for Strongly Damped Wave Equations. Nonlinear Analysis: Real World Applications, 10, 1097-1100. http://dx.doi.org/10.1016/j.nonrwa.2007.12.001

[8] Yang, M. and Sun, C. (2010) Exponential Attractors for the Strongly Damped Wave Equations. Nonlinear Analysis: Real World Applications, 11, 913-919. http://dx.doi.org/10.1016/j.nonrwa.2009.01.022

[9] Zhou, S. (1999) Dimension of the Global Attractor for Strongly Damped Nonlinear Wave Equation. Journal of Mathematical Analysis and Applications, 233, 102-115. http://dx.doi.org/10.1006/jmaa.1999.6269

[10] Zhou, S. and Fan, X. (2002) Kernel Sections for Non-Autonomous Strongly Damped Wave Equations. Journal of Mathematical Analysis and Applications, 275, 850-869. http://dx.doi.org/10.1016/S0022-247X(02)00437-7

[11] Zhou, S. (2003) Attractors for Strongly Damped Wave Equations with Critical Exponent. Applied Mathematics Letters, 
16, 1307-1314. http://dx.doi.org/10.1016/S0893-9659(03)90134-0

[12] Chow, P. (2002) Stochastic Wave Equation with Polynomial Nonlinearity. Annals of Applied Probability, 12, $361-381$. http://dx.doi.org/10.1214/aoap/1015961168

[13] Fan, X. (2004) Random Attractor for a Damped Sine-Gordon Equation with White Noise. Pacific Journal of Mathematics, 216, 63-76. http://dx.doi.org/10.2140/pjm.2004.216.63

[14] Fan, X. and Wang, Y. (2007) Fractal Dimensional of Attractors for a Stochastic Wave Equation with Nonlinear Damping and White Noise. Stochastic Analysis and Applications, 25, 381-396. http://dx.doi.org/10.1080/07362990601139602

[15] Fan, X. (2008) Random Attractors for Damped Stochastic Wave Equations with Multiplicative Noise. International Journal of Mathematics, 19, 421-437. http://dx.doi.org/10.1142/S0129167X08004741

[16] Fan, X. (2006) Attractors for a Damped Stochastic Wave Equation of Sine-Gordon Type with Sublinear Multiplicative Noise. Stochastic Analysis and Applications, 24, 767-793. http://dx.doi.org/10.1080/07362990600751860

[17] Jones, R. and Wang, B. (2013) Asymptotic Behavior of a Class of Stochastic Nonlinear Wave Equations with Dispersive and Dissipative Terms. Nonlinear Analysis: Real World Applications, 14, 1308-1322. http://dx.doi.org/10.1016/j.nonrwa.2012.09.019

[18] Lu, K. and Schmalfuß, B. (2007) Invariant Manifolds for Stochastic Wave Equations. Journal of Differential Equations, 236, 460-492. http://dx.doi.org/10.1016/j.jde.2006.09.024

[19] Lv, Y. and Wang, W. (2008) Limiting Dynamics for Stochastic Wave Equations. Journal of Differential Equations, 244, 1-23. http://dx.doi.org/10.1016/j.jde.2007.10.009

[20] Wang, B.X. and Gao, X.L. (2009) Random Attractors for Wave Equations on Unbounded Domains. Discrete and Continuous Dynamical Systems, Special, 800-809.

[21] Wang, B. (2011) Asymptotic Behavior of Stochastic Wave Equations with Critical Exponents on $\mathbb{R}^{3}$. Transactions of the American Mathematical Society, 363, 3639-3663. http://dx.doi.org/10.1090/S0002-9947-2011-05247-5

[22] Wang, Z., Zhou, S. and Gu, A. (2012) Random Attractor of the Stochastic Strongly Damped Wave Equation. Communications in Nonlinear Science and Numerical Simulation, 17, 1649-1658. http://dx.doi.org/10.1016/j.cnsns.2011.09.001

[23] Wang, Z., Zhou, S. and Gu, A. (2011) Random Attractor for a Stochastic Damped Wave Equation with Multiplicative Noise on Unbounded Domains. Nonlinear Analysis: Real World Applications, 12, 3468-3482. http://dx.doi.org/10.1016/j.nonrwa.2011.06.008

[24] Yang, M., Duan, J. and Kloeden, P. (2011) Asymptotic Behavior of Solutions for Random Wave Equations with Nonlinear Damping and White Noise. Nonlinear Analysis: Real World Applications, 12, 464-478. http://dx.doi.org/10.1016/j.nonrwa.2010.06.032

[25] Zhou, S., Yin, F. and Ou Yang, Z. (2005) Random Attractor for Damped Nonlinear Wave Equations with White Noise. The SIAM Journal on Applied Dynamical Systems, 4, 883-903. http://dx.doi.org/10.1137/050623097

[26] Crauel, H. (2002) Random Probability Measure on Polish Spaces. Taylor \& Francis, London.

[27] Crauel, H., Debussche, A. and Flandoli, F. (1997) Random Attractors. Journal of Dynamics and Differential Equations, 9, 307-341. http://dx.doi.org/10.1007/BF02219225

[28] Crauel, H. and Flandoli, F. (1994) Attractors for Random Dynamical Systems. Probability Theory and Related Fields, 100, 365-393. http://dx.doi.org/10.1007/BF01193705

[29] Flandoli, F. and Schmalfuß, B. (1996) Random Attractors for the 3D Stochastic Navier-Stokes Equation with Multiplicative Noise. Stochastics and Stochastic Reports, 59, 21-45. http://dx.doi.org/10.1080/17442509608834083

[30] Shen, Z., Zhou, S. and Shen, W. (2010) One-Dimensional Random Attractor and Rotation Number of the Stochastic Damped Sine-Gordon Equation. Journal of Differential Equations, 248, 1432-1457. http://dx.doi.org/10.1016/j.jde.2009.10.007

[31] Ball, J.M. (1997) Continuity Properties and Global Attractors of Generalized Semiflows and the Navier-Stokes Equations. Journal of Nonlinear Science, 7, 475-502. http://dx.doi.org/10.1007/s003329900037

[32] Ball, J.M. (2004) Global Attractors for Damped Semilinear Wave Equations. Discrete and Continuous Dynamical Systems, 10, 31-52. http://dx.doi.org/10.3934/dcds.2004.10.31

[33] Ghidaglia, J.M. (1994) A Note on the Strong Convergence towards Attractors for Damped Forced KdV Equations. Journal of Differential Equations, 110, 356-359. http://dx.doi.org/10.1006/jdeq.1994.1071

[34] Goubet, O. and Rosa, R. (2002) Asymptotic Smoothing and the Global Attractor of a Weakly Damped KdV Equation on the Real Line. Journal of Differential Equations, 185, 25-53. http://dx.doi.org/10.1006/jdeq.2001.4163

[35] Ju, N. (2000) The $H^{1}$-Compact Global Attractor for the Solutions to the Navier-Stokes Equations in Two-Dimensional 
Unbounded Domains. Nonlinearity, 13, 1227-1238. http://dx.doi.org/10.1088/0951-7715/13/4/313

[36] Moise, I. and Rosa, R. (1997) On the Regularity of the Global Attractor of a Weakly Damped, Forced Korteweg-de Vries Equation. Advances in Differential Equations, 2, 257-296.

[37] Moise, I., Rosa, R. and Wang, X. (1998) Attractors for Non-Compact Semigroups via Energy Equations. Nonlinearity, 11, 1369-1393. http://dx.doi.org/10.1088/0951-7715/11/5/012

[38] Rosa, R. (1998) The Global Attractor for the 2D Navier-Stokes Flow on Some Unbounded Domains. Nonlinear Analysis, 32, 71-85. http://dx.doi.org/10.1016/S0362-546X(97)00453-7

[39] Wang, X. (1995) An Energy Equation for the Weakly Damped Driven Nonlinear Schrodinger Equations and Its Applications. Physica D, 88, 167-175. http://dx.doi.org/10.1016/0167-2789(95)00196-B

[40] Bates, P.W., Lu, K. and Wang, B. (2009) Random Attractors for Stochastic Reaction-Diffusion Equations on Unbounded Domains. Journal of Differential Equations, 246, 845-869. http://dx.doi.org/10.1016/j.jde.2008.05.017

[41] Chueshov, I. (2002) Monotone Random Systems Theory and Applications. Springer-Verlag, New York. http://dx.doi.org/10.1007/b83277

[42] Pazy, A. (1983) Semigroup of Linear Operators and Applications to Partial Differential Equations. Springer-Verlag, New York. http://dx.doi.org/10.1007/978-1-4612-5561-1 